\title{
Rrp1 translocase and ubiquitin ligase activities restrict the genome destabilising effects of Rad51 in fission yeast
}

\author{
Jakub Muraszko', Karol Kramarz ${ }^{2,3}$, Bilge Argunhan ${ }^{4}$, Kentaro Ito ${ }^{4}$, Gabriela Baranowska ${ }^{1}$, \\ Yumiko Kurokawa4, Yasuto Murayama4, Hideo Tsubouchi ${ }^{4}$, Sarah Lambert ${ }^{2,3}$,

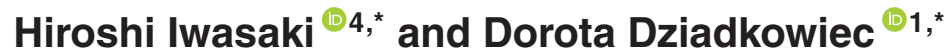

${ }^{1}$ Faculty of Biotechnology, University of Wrocław, Poland, ${ }^{2}$ Institut Curie, Université PSL, CNRS UMR3348, 91400 Orsay, France, ${ }^{3}$ Université Paris-Saclay, CNRS UMR3348, 91400 Orsay, France and ${ }^{4}$ Cell Biology Center, Institute of Innovative Research, Tokyo Institute of Technology, Japan

Received June 23, 2020; Revised May 27, 2021; Editorial Decision May 31, 2021; Accepted June 06, 2021

\section{ABSTRACT}

Rad51 is the key protein in homologous recombination that plays important roles during DNA replication and repair. Auxiliary factors regulate Rad51 activity to facilitate productive recombination, and prevent inappropriate, untimely or excessive events, which could lead to genome instability. Previous genetic analyses identified a function for Rrp1 (a member of the Rad5/16-like group of SWI2/SNF2 translocases) in modulating Rad51 function, shared with the Rad51 mediator Swi5-Sfr1 and the Srs2 anti-recombinase. Here, we show that Rrp1 overproduction alleviates the toxicity associated with excessive Rad51 levels in a manner dependent on Rrp1 ATPase domain. Purified Rrp1 binds to DNA and has a DNA-dependent ATPase activity. Importantly, Rrp1 directly interacts with Rad51 and removes it from double-stranded DNA, confirming that Rrp1 is a translocase capable of modulating Rad51 function. Rrp1 affects Rad51 binding at centromeres. Additionally, we demonstrate in vivo and in vitro that Rrp1 possesses E3 ubiquitin ligase activity with Rad51 as a substrate, suggesting that Rrp1 regulates Rad51 in a multi-tiered fashion.

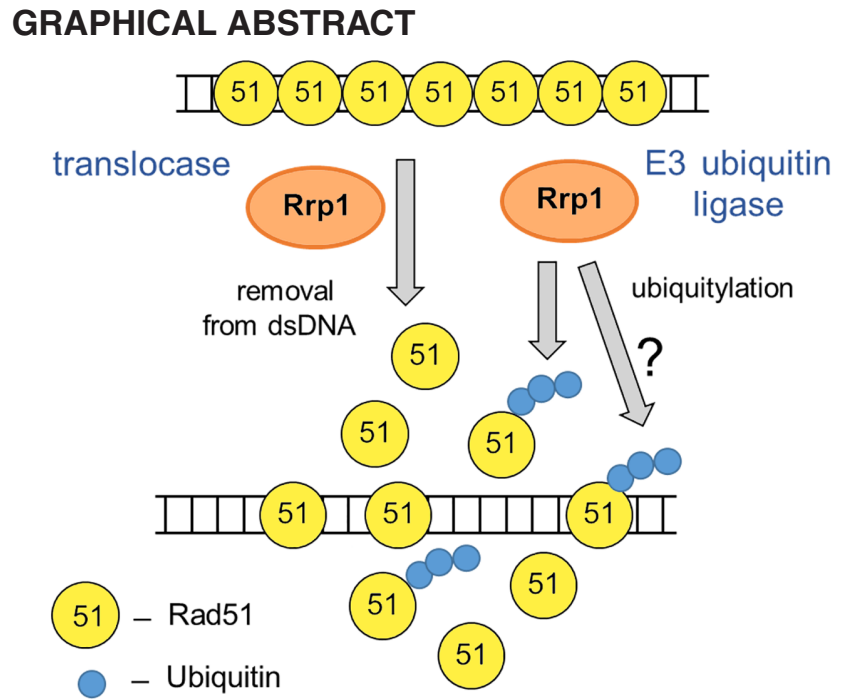

\section{INTRODUCTION}

Homologous recombination (HR) is a highly conserved pathway for the repair of DNA double-strand breaks (DSBs), and many key HR proteins have a critical role during DNA replication (1). During DSB repair, the Rad51 recombinase forms a nucleoprotein filament on singlestranded DNA (ssDNA) that catalyses strand invasion into intact homologous double-stranded DNA (dsDNA) $(2,3)$. $\operatorname{Rad} 51$ is aided by a group of proteins called recombination mediators. The main mediator in yeasts, Rad52, facilitates Rad51 loading onto replication protein A (RPA)-coated ssDNA $(4,5)$. In human cells, the tumour suppressor protein

\footnotetext{
*To whom correspondence should be addressed. Tel: +48 71375 6238; Fax: +48 71375 6238; Email: dorota.dziadkowiec@uwr.edu.pl

Correspondence may also be addressed to Hiroshi Iwasaki. Email: hiwasaki@bio.titech.ac.jp

Present addresses:

Bilge Argunhan, Section of Structural Biology, Faculty of Medicine, Imperial College London, UK.

Yumiko Kurokawa and Yasuto Murayama, Center for Frontier Research, National Institute of Genetics, Japan. 
BRCA2 fulfils an equivalent function during HR, recruiting Rad51 onto RPA-coated ssDNA and stabilising presynaptic filaments (6). Additionally, human cells contain five canonical Rad51 paralogs (RAD51B, RAD51C, RAD51D, $\mathrm{XRCC} 2$ and XRCC3) that influence HR, and these factors are thought to stimulate Rad51 activity, reviewed in (7). In the fission yeast Schizosaccharomyces pombe, three auxiliary factor complexes have been shown to promote Rad51-dependent DNA repair: Sws1-Rlp1-Rd11, Rad55Rad57 and Swi5-Sfr1, all of which are conserved in humans (8-11). We previously identified another complex, Rrp1Rrp2, that acts in a Swi5-Sfr1-dependent sub-pathway of HR in the replication stress response and modulates Rad51 activity $(12,13)$. Rrp1 and Rrp2 also have distinct roles in modulating histone dynamics that affect centromere stability (14). Additionally, Rrp2 has been shown to protect cells from Top2-induced DNA damage (15) and to play a role in telomere maintenance (14) independently of Rrp1.

Replication forks frequently stall at specific sites in the genome, such as repetitive DNA sequences, DNA lesions resulting from exogenous damage, or sites of DNA-protein association (16), and RAD51 has multiple important functions at such arrested forks. First, RAD51 binding stabilizes replication forks by protecting them from nucleolytic degradation $(17,18)$. Second, RAD51 participates in replication fork reversal, a global mechanism to stabilise forks and protect them from breakage, and stimulates the fork regression activity of RAD54, a SWI2/SNF2-like translocase, by inhibiting fork restoration $(19,20)$. Several SNF2family DNA translocases, such as SMARCAL1, ZRANB3 and HLTF, are able to remodel replication forks (21-24), and RAD51 is proposed to cooperate in this process by driving the equilibrium of the reaction toward fork reversal (25). Finally, when the replication fork is inactivated or converted to a DSB by MUS81-dependent nucleolytic cleavage, the strand exchange activity of RAD51 promotes HRdependent reconstitution of replication (26-28).

RAD51 filament formation must be tightly regulated because inappropriate, excessive, or untimely recombination (especially at replication forks or repeated sequences) can lead to deleterious effects including loss of heterozygosity and chromosome rearrangements that are hallmarks of cancer in higher organisms (29). Many helicases, such as Sgs1 and Srs2 in yeast, as well as BLM, PARI, FANCJ and RECQ5 in mammals, have been implicated in regulating the stability of RAD51 filaments formed on ssDNA. This ensures that the HR-mediated DSB repair process is reversible and can proceed along multiple pathways, making it both flexible and robust, reviewed in (30). Recently, RADX has been found to antagonise RAD51 binding to ssDNA specifically at replication forks, where it regulates the balance between RAD51 fork protection, fork reversal and its role in DSB repair $(31,32)$.

Rad51 must not only be able to form filaments on ssDNA but also bind to dsDNA tracts in order to carry out its multiple functions, and this process is also stringently regulated. The SWI2/SNF2-like translocase Rad54 dissociates Rad51 from dsDNA in both yeast and human cells (33-35), allowing for the repair synthesis by DNA polymerases that is necessary for the completion of HR. Rad54 is activated in G2 and does not remove Rad51 from stalled replication forks
(36). Another complex, MMS22L-TONSL, has been shown in human cells to limit RAD51 binding to dsDNA and stimulate HR-mediated restart of arrested replication forks (37). Importantly, other SWI2/SNF2-like translocases, namely Rdh54 and Uls1, cooperate with Rad54 in Saccharomyces cerevisiae not only to antagonise Rad51 binding to dsDNA during HR, but also to counteract its toxic accumulation on undamaged chromatin (38-40). RAD54L and RAD54B in humans also prevent the genome-destabilising consequences of excessive RAD51 binding to dsDNA (35). It should also be noted that the binding of RAD51 to dsDNA renders the dsDNA inaccessible to the RAD51-ssDNA filament and thus acts as a barrier to HR itself (41).

Interestingly, the RAD51 paralog RAD51C has been shown to prevent proteasomal degradation of RAD51 in human cells, especially after DNA damage (42), suggesting that RAD51 can also be regulated by ubiquitylation. Recently, RAD51 was found to be poly-ubiquitylated by the E3 ubiquitin ligase RFWD3 in a process stimulated by DNA damage (43). RAD51 ubiquitylation decreases RAD51 binding to ssDNA and leads to its proteasomal degradation, while also stimulating chromatin loading of RAD54. It has therefore been proposed that RAD51 ubiquitylation promotes its removal from sites of DNA damage and is necessary for completion of HR DNA repair (43).

Regulation of Rad51 by Fbh1, an F-box helicase and E3 ubiquitin ligase, is more complex and involves both activities of this protein. In $S$. pombe, Fbhl acts as a translocase and disrupts Rad51 ssDNA filaments, thereby regulating the outcome of the HR reaction. Additionally, the $\mathrm{SCF}^{\mathrm{Fbh} 1} \mathrm{E} 3$ complex can ubiquitylate Rad51 in vitro and this modification was found to be necessary for depletion of Rad51 in stationary-phase cells (44). Human RAD51 is also monoubiquitylated in vitro by $\mathrm{SCF}^{\mathrm{BBH} 1}$ but this does not result in the protein's turnover by proteolysis (45). Instead, the authors propose that during replication stress, FBH1 translocase displaces RAD51 from ssDNA and modifies it to prevent its reloading, thus restricting untimely HR at the replication fork.

Many proteins with helicase, translocase and/or ubiquitin ligase activities have been found to regulate Rad51 activity, underscoring the importance of this process. Here, we show that $S$. pombe Rrp1, an orthologue of $S$. cerevisiae Uls1, belonging to the unique RING-domain-containing Rad5/16-like group of SWI2/SNF2 translocases, affects $\operatorname{Rad} 51$ binding at centromeres, ubiquitylates $\operatorname{Rad} 51$ and is able to displace it from dsDNA. We propose that these translocase and ubiquitin ligase activities allow Rrp1 to counteract the genotoxic effects of excessive Rad51 binding to specific regions of chromatin, such as centromeric regions.

\section{MATERIALS AND METHODS}

\section{Yeast strains, plasmids and general methods}

Strains, plasmids and primers used are listed in Supplementary Tables S1, S2 and S3, respectively.

Media used for $S$. pombe growth were as described (46). Yeast cells were grown at $28^{\circ} \mathrm{C}$ in complete yeast extract plus supplements (YES) medium or glutamate supplemented 
Edinburgh minimal medium (EMM). Where required, thiamine was added at $5 \mu \mathrm{g} / \mathrm{ml}$ and geneticin (ICN Biomedicals) at $100 \mu \mathrm{g} / \mathrm{ml}$. Strains were obtained by classical yeast genetics techniques. pREP81-FLAG vector and plasmids carrying wild-type and mutated forms of $r r p 1^{+}$and $r r p 2^{+}$, as well as domains of the $\mathrm{rrpl}^{+}$gene used in the yeast two hybrid system, were constructed using the Gibson Assembly ${ }^{\circledR}$ Cloning Kit (NEB). Amplified fragments were cloned into NdeI and BamHI digested pREP81 vector. After Gibson cloning, inserts were cut by NdeI and SmaI digestion and cloned into pREP42-HA, pREP42-EGFP, pREP41mCherry, or pGADT7 and pGBKT7 plasmids. rrpl $^{+}$and rrp1-CS mutant version were introduced into the pGEX vector (GE Healthcare) by In-Fusion ${ }^{\circledR}$ cloning (Takara Bio). All constructs were checked by sequencing.

\section{Spot assays}

Cells were grown to mid-log phase, then serially diluted 10fold, and $2 \mu \mathrm{l}$ aliquots were spotted onto relevant plates (YES or EMM) that were incubated for $3-5$ days at $28^{\circ} \mathrm{C}$ and photographed. All assays were repeated at least twice.

\section{Survival assay}

Cells were grown for $48 \mathrm{~h}$ in YES or in minimal medium with (repressed conditions) or without thiamine (overexpression) at $28^{\circ} \mathrm{C} .500 \mu \mathrm{l}$ aliquots were collected, serially diluted, plated onto YES plates and incubated for 3-5 days at $28^{\circ} \mathrm{C}$. The viable cells were counted and percentage of survival for gene overexpression conditions was calculated against the repressed control.

\section{Yeast two-hybrid assay}

Gal4-based Matchmaker Two-Hybrid System 3 (Clontech) was used. The indicated proteins were fused to the GAL4 activation domain (AD) in pGADT7 vector and the GAL4 DNA-binding domain (DBD) in pGBKT7, and expressed in the $S$. cerevisiae tester strain AH109. Transformants were selected on synthetic dextrose drop-out medium without Leu and Trp (SD DO-2), and then plated on low stringency medium without Leu, Trp and His (SD DO-3) and high stringency medium without Leu, Trp, His and Ade (SD DO4 ), and incubated for $3-5$ days at $28^{\circ} \mathrm{C}$.

\section{Fluorescence microscopy}

To determine the formation of Rrpl foci and their colocalisation with Rad51 foci, appropriate transformants were grown for $24 \mathrm{~h}$ in EMM medium without thiamine. $1 \mathrm{ml}$ of culture was harvested, washed with water and subjected to fluorescent microscopy analysis. For co-localisation experiments, data were collected under $63 \times$ magnification with the confocal microscope Leica 453 TCS SP8 (Leica Microsystems) equipped with Leica HyD SP detector, and analysed with LAS X 3.3.0. For examination of mitotic defects induced by $\mathrm{rad} 5 \mathrm{I}^{+}$overexpression, samples taken from respective transformant cultures grown for $48 \mathrm{~h}$ in EMM medium without thiamine were washed and fixed in $70 \%$ ethanol. After rehydration, cells were stained with 1 $\mathrm{mg} / \mathrm{ml}$ 4',6-diamidino-2-phenylindole (DAPI) and $1 \mathrm{mg} / \mathrm{ml}$ $p$-phenylenediamine in $50 \%$ glycerol and examined by fluorescence microscopy with Axio Imager A.2 (Carl Zeiss).

\section{Rad51 foci detection by immunofluorescence}

Strains grown in complete EMM media were fixed with formaldehyde at 4\% final concentration for $45 \mathrm{~min}$ with shaking at room temperature then washed with PBS and subsequently with PEM buffer (100 mM PIPES, $1 \mathrm{mM}$ EGTA, $1 \mathrm{mM} \mathrm{MgSO}_{4}, \mathrm{pH}$ 6.9). Next, the cell wall was digested for $10 \mathrm{~min}$ at $30^{\circ} \mathrm{C}$ with $100 \mathrm{~T}$ zymolyase (MP Biomedicals, SKU08320932) at a final concentration of 0.5 $\mu \mathrm{g} / \mathrm{ml}$ in PEMS (PEM with 1.2 M sorbitol). After three washes with PEMS, cells were treated with $1 \%$ triton X-100 in PEMS for $5 \mathrm{~min}$ at RT. Next, cells were washed twice with PEMBAL (1\% BSA, 0.1\% sodium azide, $100 \mathrm{mM}$ lysine monohydrate (Sigma L-5626) in PEM buffer) and incubated on the wheel for $1 \mathrm{~h}$ in PEMBAL. Subsequently, cells were resuspended in $300 \mu \mathrm{l}$ of PEMBAL with anti-Rad51 antibody (Abcam, ab63799, 1:300) overnight on a wheel at room temperature. After washes with PEMBAL buffer, two of $10 \mathrm{~min}$ each and a third for $30 \mathrm{~min}$ on a wheel, the cells were resuspended in $300 \mu \mathrm{l}$ of PEMBAL with anti-Rabbit Alexa Fluor 555 (Molecular Probes, A21428, 1:1000) for $2 \mathrm{~h}$ at room temperature. After three washes in PBS, each for 10 min, cells were resuspended in $1 \mathrm{ml}$ of PBS with DAPI (diluted 1:4000) for $3 \mathrm{~min}$. Then cells were washed with PBS to remove excess DAPI. Finally, cells were resuspended in 10 $\mu \mathrm{l}$ of ProLong Gold antifade reagent (Invitrogen, P36934) and subjected to snapshot microscopy on glass slides using a 3D LEICA DMRXA microscope, supplied with CoolSNAP monochromic camera (Roper Scientific) under $100 \times$ oil immersion magnification with numerical aperture 1.4. In all, $14 \mathrm{z}$-stack pictures of $300 \mathrm{~nm}$ with $400 \mathrm{~ms}$ exposure for Alexa 555 and $50 \mathrm{~ms}$ for DAPI channels were collected with METAMORPH and analyzed with ImageJ Software.

\section{Chromatin immunoprecipitation (ChIP) of Rad51}

ChIP against Rad51 was performed as described in (47) with the following modifications. $100 \mathrm{ml}$ of logarithmic cell cultures with thiamine (RTS1-RFB OFF, inactive replication fork block on chromosome III) or without thiamine (RTS1-RFB ON, active replication fork block on chromosome III) were crosslinked with $10 \mathrm{mM}$ DMA (dimethyl adipimidate, Thermo Scientific, 20660) and then with $1 \%$ formaldehyde (Sigma, F-8775). Next, cells were frozen in liquid nitrogen and lysed by bead beating in $400 \mu \mathrm{l}$ of lysis buffer (50 mM HEPES pH 7.5, 1\% Triton X100, 0.1\% sodium deoxycholate, $1 \mathrm{mM}$ EDTA, $1 \mathrm{mM}$ PMSF and Complete EDTA-free protease inhibitor cocktail tablets (Roche, 187358). After that, chromatin sonication was performed using Diagenode Bioruptor, 10 cycles of $30 \mathrm{~s} \mathrm{ON}$ and $30 \mathrm{~s} \mathrm{OFF}$ at $4{ }^{\circ} \mathrm{C}$. Immunoprecipitation was done overnight with an anti-Rad51 antibody (Abcam, ab63799, 1:300). Protein G Dynabeads (Invitrogen, 10003D) were then added for $1 \mathrm{~h}$ and crosslinking was reversed by incubating the sample at $65^{\circ} \mathrm{C}$ for $2 \mathrm{~h}$. DNA associated with Rad51 was purified with Qiaquick PCR purification kit (QUIAGEN, 28104) and eluted in $400 \mu \mathrm{l}$ of water. qPCR 
(iQ SYBR green supermix, Biorad, 1708882, primers listed in Supplementary Table S3) was performed to determine the relative amounts of DNA (starting quantities based on standard curves for each pair of primers) using BIORAD CFX Maestro v1.1. The enrichment at each locus was determined by subtracting the negative control values ( $\mathrm{rad} 51 \Delta$ strain) and internal control locus at chromosome II (named II.50).

\section{In vivo co-immunoprecipitation}

In vivo pull-down experiments were performed using strains with native levels of Rad51 and overproduction of FLAGtagged Rrp1 or Rrp2 under the control of the low strength $n m t 81$ promotor. Cells were grown to mid-log phase using EMM minimal medium for $24 \mathrm{~h} .100 \mathrm{ml}$ of cells were harvested and broken with glass beads in $\mathrm{H}$ buffer $(50$ $\mathrm{mM}$ HEPES-KOH pH 7, $50 \mathrm{mM}$ KOAc, $5 \mathrm{mM}$ MgOAc, $0.1 \%$ NP-40, $10 \%$ glycerol, $1 \mathrm{mM}$ DTT and $1 \times \mathrm{cOm}-$ plete $^{T M}$ EDTA-free protease inhibitor cocktail (Roche)). Extracts were cleared by centrifugation and immunoprecipitated with ANTI-FLAG ${ }^{\circledR}$ M2 Affinity Gel (Sigma). Beads were washed and eluted using $100 \mu \mathrm{g} / \mathrm{ml}$ 3xFLAG peptide (Sigma). For detection, anti-FLAG (1:5000, Sigma) antibodies and anti-Rad51 (1:5000, (48)) antiserum were used.

\section{Purification of Rrp1-FLAG}

Recombinant Rrp1 and Rrp1-CS were expressed in the Rosetta E. coli strain (Novagen) from the pGEX-6P plasmid (GE Healthcare). Proteins were C-terminally fused to the GST tag and N-terminally to the 3xFLAG tag; only the former was removed during the purification process. Expression was induced with $1 \mathrm{mM}$ IPTG (Sigma) at $18^{\circ} \mathrm{C}$ overnight. Cells were collected by centrifugation, resuspended in R buffer (20 mM Tris, $\mathrm{pH} 7.5,10 \%$ glycerol, $1 \mathrm{mM}$ EDTA) containing $500 \mathrm{mM} \mathrm{NaCl}$, and disrupted by sonication. The cell lysate was then clarified by ultracentrifugation $\left(70000 \mathrm{~g}, 1 \mathrm{~h}, 2^{\circ} \mathrm{C}\right)$. The supernatant was mixed with 4B GSH sepharose (Sigma) for $3 \mathrm{~h}$ at $4^{\circ} \mathrm{C}$. Resin-bound proteins were eluted in R buffer containing $300 \mathrm{mM} \mathrm{NaCl}$ and $40 \mathrm{mM}$ glutathione (Sigma). The sample was supplemented with $0.2 \mu \mathrm{g} / \mathrm{ml}$ of HRV-3C protease (Sigma) to remove the GST tag and dialyzed against $\mathrm{R}$ buffer containing $100 \mathrm{mM}$ $\mathrm{NaCl}$ (overnight, $4^{\circ} \mathrm{C}$ ). The dialyzed sample was loaded onto a $1 \mathrm{ml}$ HiTrap Heparin (Sigma) column. Rrp1 eluted at around $650 \mathrm{mM} \mathrm{NaCl}$ with a linear gradient of $0.1-1.0 \mathrm{M}$ $\mathrm{NaCl}$ in $\mathrm{R}$ buffer. Eluted fractions were diluted 6.5-fold with $\mathrm{R}$ buffer and loaded onto a $1 \mathrm{ml}$ Resource Q column (GE Healthcare). Rrpl eluted at around $300 \mathrm{mM} \mathrm{NaCl}$ with a linear gradient of $0.1-1.0 \mathrm{M} \mathrm{NaCl}$ in $\mathrm{R}$ buffer. Eluted fractions were diluted 3-fold with $\mathrm{R}$ buffer and loaded onto a HiTrap SP column (GE Healthcare). Rrp1 eluted at around $600 \mathrm{mM} \mathrm{NaCl}$ with a linear gradient of $0.1-1.0 \mathrm{M} \mathrm{NaCl}$ in $\mathrm{R}$ buffer. Eluted fractions were collected and dialysed against $\mathrm{R}$ buffer containing $200 \mathrm{mM} \mathrm{NaCl}$. Concentration was determined using NanoDrop (ThermoFisher) with a molar extinction coefficient of 100365 . For the Rrp1-CS mutant, Resource Q and HiTrap SP columns were omitted. Rad51 was purified exactly as previously described (49). Uba1 (E1) and Ubc4 (E2) were purified exactly as previously described (44). All proteins were free of nuclease and/or protease activities for the duration of the relevant assays.

\section{In vitro co-immunoprecipitation}

in vitro co-immunoprecipitation assays were performed as follows. Briefly, purified Rad51 and FLAG-tagged Rrp1 (250 $\mathrm{nM}$ each) were incubated in IP buffer $(35 \mathrm{mM}$ Tris$\mathrm{HCl} \mathrm{pH}$ 7.5, $1 \mathrm{mM}$ DTT, $100 \mathrm{mM} \mathrm{NaCl}, 3.5 \mathrm{mM} \mathrm{MgCl}{ }_{2}$, $0.1 \% \mathrm{NP}-40,5 \%$ glycerol) for $30 \mathrm{~min}$ at $30^{\circ} \mathrm{C}$. Proteins were immunoprecipitated using ANTI-FLAG ${ }^{\circledR}$ M2 Affinity Gel (Sigma) for $2 \mathrm{~h}$ at $4^{\circ} \mathrm{C}$. Beads were washed three times with IP buffer, and proteins were eluted using $100 \mu \mathrm{g} / \mathrm{ml} 3 \mathrm{x}$ FLAG peptide (Sigma). Eluates were analysed by western blotting with anti-FLAG antibodies (1:5000, Sigma) and anti-Rad51 (1:5000, (48)) antiserum.

\section{Colorimetric ATPase assay}

Reaction mixtures $(22.5 \mu \mathrm{l})$ in ATPase buffer $(25 \mathrm{mM}$ Tris$\mathrm{HCl}$ [pH 7.5], $1 \mathrm{mM}$ DTT, $20 \mathrm{mM} \mathrm{NaCl}, 5 \mathrm{mM} \mathrm{MgCl} 2$ and $2 \%$ glycerol) with $30 \mathrm{nM}$ Rrpl and containing $5 \mu \mathrm{M}$ nt ssDNA, $10 \mu \mathrm{M}$ nt dsDNA or no DNA were prepared on ice. The reactions were initiated by the addition of $2.5 \mu \mathrm{l}$ of $10 \mathrm{mM}$ ATP (final concentration of $1 \mathrm{mM}$ ) and incubated at $30^{\circ} \mathrm{C}$. Aliquots $(10 \mu \mathrm{l})$ taken at time 0 and 15 min were mixed with $2 \mu 1$ of $120 \mathrm{mM}$ EDTA to stop the reaction. Inorganic phosphate generated by ATP hydrolysis was detected using the Malachite Green Phosphate Assay Kit (BioAssay Systems, USA). The ssDNA used in this assay was $16 \mathrm{~A}(-)$, an 83-mer oligo (5'-AAATGAACAT AAAGTAAATA AGTATAAGGA TAATACAAAA TAAGTAAATG AATAAACATA GAAAATAAAG TAAAGGATAT AAA-3') (50). The dsDNA used was prepared by annealing of $16 \mathrm{~A}(-)$ and its complementary 83-mer ssDNA, 16A(+).

\section{Electrophoretic mobility shift assay (EMSA)}

Purified Rrp1 was incubated with cssDNA (circular singlestrand DNA of Phi X174 viral DNA, NEB), ldsDNA (linear double-stranded DNA obtained by digesting Phi 174 RF I with ApaLI, NEB) or cccDNA (covalently closedcircular DNA of Phi 174 RF I, NEB) at $0.5 \mu \mathrm{M}$ (base pair concentration) in E buffer (25 mM HEPES pH 7.5, $1 \mathrm{mM}$ DTT, $60 \mathrm{mM} \mathrm{KCl,} 2 \mathrm{mM}$ ATP, $3.5 \mathrm{mM} \mathrm{MgCl}_{2}, 5 \%$ glycerol) for $15 \mathrm{~min}$ in $37^{\circ} \mathrm{C}$. Samples were then crosslinked with $0.2 \%$ glutaraldehyde $\left(37^{\circ} \mathrm{C}, 5 \mathrm{~min}\right)$ then run on a $0.8 \%$ agarose 1xTAE gel and stained with SYBR Gold (ThermoFisher).

\section{In vitro ubiquitylation assay}

Reactions were performed in ubiquitylation buffer $(25 \mathrm{mM}$ Tris- $\mathrm{HCl}$ [pH 7.5], $1 \mathrm{mM}$ DTT, $20 \mathrm{mM} \mathrm{NaCl}, 5 \mathrm{mM}$ ATP, $5 \mathrm{mM} \mathrm{MgCl}_{2}$ and $2 \%$ glycerol) containing $5 \mu \mathrm{M} \mathrm{S}$. cerevisiae Ubiquitin (Funakoshi, U-100SC-05M), $0.2 \mu \mathrm{M}$ HisUba1 (E1), $2 \mu \mathrm{M}$ His-Ubc4 (E2), 0.2 $\mu$ M Rrp1-FLAG (E3) and $1 \mu \mathrm{M}$ Rad51. This mixture was incubated at $37^{\circ} \mathrm{C}$ for $30 \mathrm{~min}$. When the effect of DNA was examined, $1 \mu \mathrm{M}$ Rad51 was preincubated with increasing concentrations of ldsDNA (PhiX RFI linearised with ApaLI) at $37^{\circ} \mathrm{C}$ for $10 \mathrm{~min}$ in ubiquitylation buffer supplemented with $50 \mathrm{mM}$ $\mathrm{NaCl}$ prior to the addition of ubiquitylation reaction components. Reactions were stopped by addition of $6 \times \operatorname{SDS}$ 
Laemmli buffer and proteins were resolved by $15 \%$ SDSPAGE and analysed by western blotting with anti-ubiquitin antibodies (1:2000 Abcam P4D1) and anti-Rad51 (1:5000) (48) antiserum.

\section{Rad51 removal from DNA}

EMSA-based analysis was performed as described above except that $\operatorname{Rad} 51$ was first incubated with the DNA at $37^{\circ} \mathrm{C}$ for $5 \mathrm{~min}$, then Rrp1 was added. For the measurements with ssDNA, Rad51 filaments were formed by incubation of $0.5 \mu \mathrm{M} \operatorname{Rad} 51$ with $1.5 \mu \mathrm{M}$ ssDNA (oligo-dT, 72-mer, base pair concentration) for $5 \mathrm{~min}$ at $37^{\circ} \mathrm{C}$ in buffer $(30 \mathrm{mM}$ HEPES pH 7.5, $1 \mathrm{mM}$ DTT, $50 \mathrm{mM} \mathrm{NaCl}, 100 \mathrm{mM} \mathrm{KCl}$, $2 \mathrm{mM}$ ATP, $8 \mathrm{mM}$ PC, $8 \mathrm{U} / \mathrm{ml} \mathrm{CPK}, 3.5 \mathrm{mM} \mathrm{MgCl} 2,2.5 \%$ glycerol). This mixture was transferred to a $0.2 \times 1.0 \mathrm{~cm}$ cuvette (Hellma Analytics) at $37^{\circ} \mathrm{C}$. The change in fluorescence anisotropy at $575 \mathrm{~nm}$ following excitation at $546 \mathrm{~nm}$ was measured for $60 \mathrm{~s}$. After that time, scavenger ssDNA (15 $\mu \mathrm{M}$ nucleotides PhiX 174 virion DNA) and the indicated concentrations of Rrp1 protein were added. Data were collected using an FP-8300 spectrofluorometer (JASCO) every second for $3 \mathrm{~min}$. For each reaction, the measurements $20 \mathrm{~s}$ before addition of scavenger DNA and Rrp1 were averaged, and the subsequent measurements were expressed relative to this averaged value.

For the measurements with dsDNA, $6 \mu \mathrm{M}$ of $\operatorname{Rad} 51$ was incubated with $3 \mu \mathrm{M}$ (base pair concentration) of dsDNA (5'-TAMRA-labeled 16A(-) annealed to $16 \mathrm{~A}(+))$ for $5 \mathrm{~min}$ at $37^{\circ} \mathrm{C}$ in buffer (30 mM HEPES pH 7.5, $1 \mathrm{mM}$ DTT, 50 $\mathrm{mM} \mathrm{NaCl}, 100 \mathrm{mM} \mathrm{KCl}, 2 \mathrm{mM}$ ATP, $8 \mathrm{mM} \mathrm{PC}, 8 \mathrm{U} / \mathrm{ml}$ CPK, $3.5 \mathrm{mM} \mathrm{MgCl}_{2}, 2.5 \%$ glycerol). This mixture was then transferred to a $1.0 \times 1.0 \mathrm{~cm}$ cuvette (Hellma Analytics) with a magnetic stirrer and maintained at $37^{\circ} \mathrm{C}$ with stirring (450 rpm). The change in fluorescence anisotropy at $575 \mathrm{~nm}$ following excitation at $546 \mathrm{~nm}$ was monitored for $60 \mathrm{~s}$. After that time, scavenger ssDNA ( $15 \mu \mathrm{M}$ nucleotides PhiX 174 virion DNA) was added, and after $60 \mathrm{~s}, 0.25 \mu \mathrm{M}$ of Rrp1 was injected into the mixture. Data were collected using an FP-8300 spectrofluorometer (JASCO) every second for over $500 \mathrm{~s}$. tion:

$\Delta$ anisotropy was calculated using the following equa-

$$
\begin{aligned}
& \Delta \text { anisotropy } \\
& =\text { raw value of (anisotropy with proteins at time } t) \\
& \quad-(\text { anisotropy without proteins at time } t)
\end{aligned}
$$

Time zero was defined as the time when Rrpl was added into the reaction mixture.

The dissociation rate $\left(k_{\text {off }}\right)$ values were obtained by fitting the time dependence of $\Delta$ anisotropy, with the following equation:

$$
\begin{aligned}
\Delta \text { anisotropy }= & (\text { Maximum value of } \Delta \text { anisotropy }) \\
& \times \exp \left(-k_{o f f} \cdot t\right)
\end{aligned}
$$

\section{Statistical data analysis}

For viability assays ANOVA test, and for ChIP assays twosided Student's $t$-test, were used to calculate the $P$-values.
To assess statistical significance of proportions of cells with aberrant mitosis and the Rad51 localisation pattern, the Ztest for two population proportions was used to calculate the $z$-statistic and corresponding $P$-values. (*** $P \leq 0.001$, ** $0.001<P \leq 0.01, * 0.01<P \leq 0.05)$.

\section{RESULTS}

\section{Rrp1 counteracts rad51 $1^{+}$overexpression-induced toxicity}

Previous studies have shown that $\mathrm{rad} 51^{+}$overexpression in $S$. pombe results in a severe growth defect (51). Rad51 overproduction leads to its excessive accumulation on chromatin and has a negative effect on cell growth and chromosome stability that is aggravated in mutants devoid of SWI2/SNF2-related translocases: RAD54L and RAD54B in humans (35), and Rdh54, Rad54 and Uls1 in S. cerevisiae (39). Two ULS1 orthologues, Rrp1 and Rrp2, have been identified in $S$. pombe (12), so we set out to examine their functional interaction with Rad51. Using an nmtP3$G F P$-rad51 strain, where the GFP-rad51 $1^{+}$gene is expressed from a strong $n m t$ promoter (nmtP3) that is induced in media lacking thiamine, we confirmed that induction of $\mathrm{rad} 51^{+}$ expression (Figure 1A, EMM plate) resulted in a severe growth defect and loss of viability (Figure 1B). Importantly, this was further exacerbated by deletion of $r r p 1^{+}$, but not $r r p 2^{+}$. Growth inhibition of the $n m t P 3-G F P$-rad51 rrpl $\Delta$ strain was visible even on media where gene expression from the $n m t P 3$ promoter is very limited (Figure 1A, YES and $\mathrm{EMM}+$ thi plates), indicating that even a mild increase in Rad51 protein levels may be toxic when Rrp1 is absent.

We thus hypothesised that if deletion of $r r p 1^{+}$is toxic in the $n m t P 3-G F P$-rad51 strain, $r r p 1^{+}$overexpression should be beneficial. Indeed, we found that overexpression of $r r p 1^{+}$, but not $r r p 2^{+}$, from the low-strength $n m t$ promoter (nmt81), rescued the growth defect (Figure 1C) and viability loss induced by GFP-Rad51 overproduction (Supplementary Figure S1A). The level of Rrp1 and Rrp2 proteins in these cells was comparable (Supplementary Figure S1B).

Overproduction of Rad51 without the GFP tag resulted in similar toxicity as GFP-Rad51 and simultaneuos overproduction of Rrp1, but not Rrp2, rescued the associated growth defect (Figure 1D) and viability loss (Supplementary Figure S1C) to the wild-type level (all genes were overexpressed from the medium-strength $n m t$ promoters $n m t 41$ or $n m t 42$ ). These results indicate that the observed phenomena are not caused by the GFP tag on Rad51, allowing us to utilise a synthetic dosage lethality approach with the nmtP3-GFP-rad51 strain to assess the effect of Rrp1 on Rad51 activity.

\section{The ATPase activity of Rrp1 is required to counter the geno- toxicity associated with Rad51 overproduction}

Rrp1 shares a complex domain structure with Uls1 (12), and contains an ATPase domain with Walker A and B motifs, characteristic for SNF2 translocases, as well as a RING domain typical for ubiquitin ligases. Walker B ( $r r p 1-D A E A)$ and RING (rrp1-CS) Rrp1 mutant variants (Figure 1E) were therefore used to examine the importance of the putative DNA translocase and ubiquitin ligase Rrp1 activities 
A

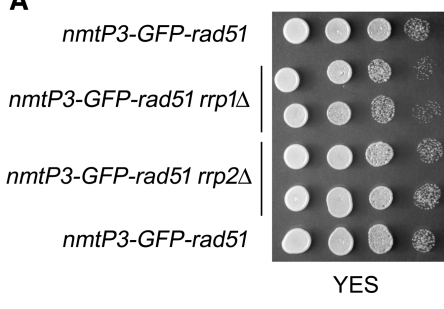

C nmtP3-GFP-rad51

+pREP81-FLAG

$+\operatorname{Rrp} 1$

+pREP81-FLAG

+ Rrp2

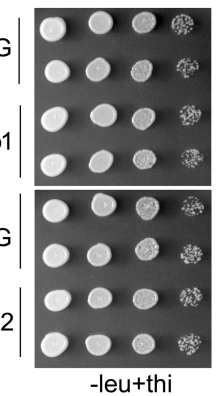

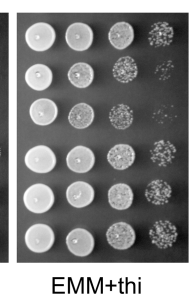

EMM+thi

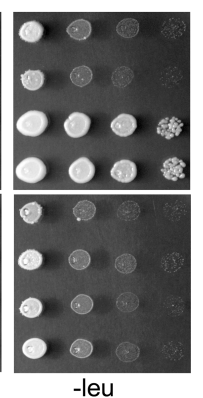

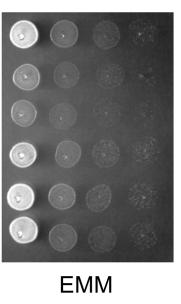

D

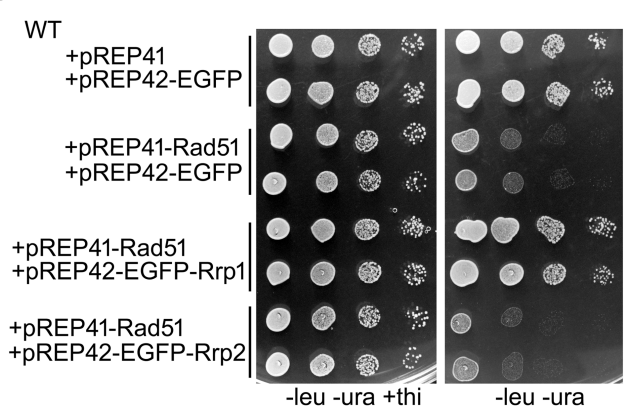

E

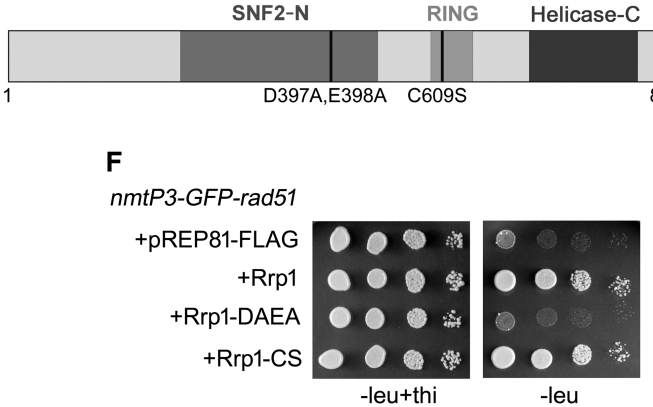

H
B

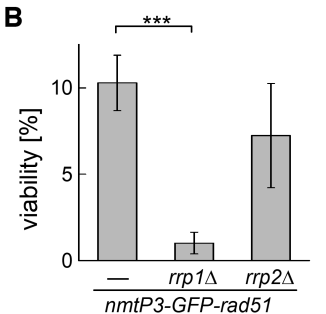

G

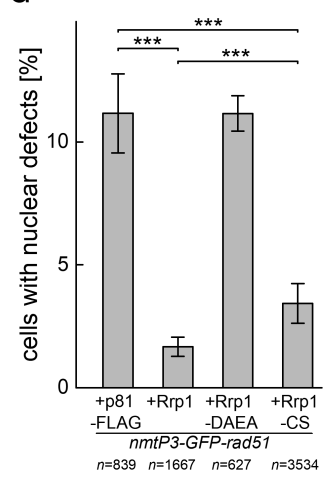

Figure 1. The ATPase activity of Rrp1 is important for viability and proper chromosome segregation in cells overexpressing rad51 $1^{+}$. (A) Deletion of $r r p 1^{+}$, but not $r r p 2^{+}$, exacerbates the growth defect induced by GFP-rad51 ${ }^{+}$overexpression. Growth of the indicated strains under conditions where $G F P$-rad $51^{+}$ overexpression was induced (EMM) or repressed (EMM + thiamine, YES control plate) was examined by spot test analysis. (B) Viability loss caused by GFP-rad51 $1^{+}$overexpression is greater in $\operatorname{rrp} 1 \Delta$ cells. Ratio of surviving cells for strains grown in the absence of thiamine (induction of expression) to those grown in its presence (without induction) was determined. The experiment was repeated for four independent cultures of each strain. ANOVA test was used to calculate $P$-values. (C) Spot test analysis demonstrating that overexpression of $r r p 1^{+}$, but not $r r p 2^{+}$, suppresses the growth defect caused by GFP-Rad51 overproduction (-leu). (D) GFP tag is not responsible for the growth defect seen in GFP-Rad51 overproducing cells and simultaneous overexpression of $r r p 1^{+}$, but not $r r p 2^{+}$, suppresses this toxicity. Wild-type cells were transformed with plasmids containing genes for Rrp1 or Rrp2 and untagged Rad51, to assess by spot test analysis the effect of their overexpression on growth (-leu-ura). Two independent transformants for each set of plasmids are shown. (E) Mutations abolishing Rrp1 putative SWI2/SNF2 DNA translocase (Walker B mutant: DAEA) and ubiquitin ligase (RING mutant: CS) activity are shown. (F) Functional Rrpl translocase is required for suppression of the growth defect in GFP-rad51 $1^{+}$overexpressing cells. Cells were transformed with plasmids harbouring genes for wild-type or mutated versions of the respective proteins and the effect of their overexpression on the growth of cells overproducing GFP-Rad51 (leu) or not (-leu+thi) was assessed by spot test analysis. (G) Accumulation of mitotic aberrations in $G F P$-rad51 $1^{+}$overexpressing cells is prevented by $\mathrm{rrpl}^{+}$overexpression and depends on the functional translocase, but not the RING domain. Cells with unequally segregated genetic material (cut and non-disjunction) were observed by DAPI staining of the nuclei of transformants grown for $48 \mathrm{~h}$ in media lacking thiamine. $n=$ total number of cells counted for 3 independent transformants of vector, $r r p 1^{+}$, rrpl-DAEA or rrpl-CS. The error bars represent the standard deviation about the mean values. The Z-test for two population proportions was used to calculate the $Z$-statistic and corresponding $P$-values. (H) Overproduction of the Rad51-L1 (F254A) mutant defective in DNA binding results in only a mild growth defect that is not rescued by overproduction of Rrp1. Wild-type cells were transformed with plasmids harbouring genes for Rrp1 and wild-type Rad51 or Rad51-L1, and the effect of their overexpression on growth (leu-ura) was assessed by spot test analysis. Two independent transformants overproducing Rad51-L1 are shown. (*** $P \leq 0.001, * * 0.001<P \leq 0.01, * 0.01<P \leq 0.05$ ). 
for counteracting GFP-rad51 $1^{+}$overexpression-induced toxicity. While the presence of a functional Rrp1 ATPase domain is required for restoration of normal growth to the GFP-rad $51^{+}$overexpressing strain, the RING domain appears to be dispensable (Figure 1F). The protein levels of Rrp1, Rrp1-DAEA and Rrp1-CS in these cells were comparable (Supplementary Figure S1D).

It has previously been shown that $\mathrm{rad} 5 \mathrm{I}^{+}$overexpression results in mitotic aberrations revealed by the accumulation of cells with nuclei exhibiting the cut (cell untimely torn) phenotype (51). We demonstrated that overproduction of Rrp1, but not the Rrp1-DAEA mutant, was able to suppress the appearance of nuclear defects, such as cut and non-disjunction, that occur in the $G F P$-rad $5 I^{+}$overexpressing strain (Figure 1G). Interestingly, the rescue induced by Rrp1-CS overproduction was less pronounced than that by wild-type Rrp1, with more cells undergoing aberrant mitosis (Figure 1G). Thus, replication stress in $\mathrm{rad}_{51} \mathrm{I}^{+}$overexpressing cells was greater when Rrp1-CS, rather than wild-type Rrp1, was overproduced although it did not reach a level that would cause a growth defect (Figure 1F). This suggests that Rrp1 ubiquitin ligase activity might also have a role in the protein's functional interaction with Rad51.

It has been proposed that excessive Rad51 binding to undamaged DNA is the cause of Rad51 overproduction toxicity in human cells (35). Indeed, we found that overproduction of the Rad51-L1 (F254A) mutant, which corresponds to human RAD51-L1 (Y232A) that is defective in DNA binding (52), resulted in only slight growth inhibition, and rrp1+ overexpression did not affect this phenotype (Figure 1H). This was not due to the lower level of Rad51-L1 than wild-type Rad51 produced from the constructs used (Supplementary Figure S1E). Taken together, these data imply that Rrp1 may counteract Rad51-overproduction induced toxicity by interfering with Rad51 binding to DNA.

\section{Rrp1 prevents excessive Rad51 accumulation on DNA}

It has been shown that endogenous Rad51 forms only few spontaneous foci in unchallenged cells (53-56). Consistently, we were able to detect such spontaneous Rad51 foci in approximately $10 \%$ of cells examined by immunofluorescence microscopy. This number increased twofold when $r r p 1^{+}$was deleted (Figure 2A). Of note, cells exhibiting $>1$ focus were also more frequently observed in the absence of Rrp1.

To address modulation of Rad51 foci formation by Rrp1 in living cells, we took advantage of the $n m t P 3-G F P$-rad51 strain cultured on YES medium, where gene expression from the $n m t P 3$ promoter is very limited, and observed that 1-3 GFP-Rad51 foci were present in about $20 \%$ of cells (Figure 2B, top middle panel). Interestingly, deletion of $r r p 1^{+}$not only led to an increase in the number of cells with one GFP-Rad51 focus but also resulted in a marked increase in the number of cells with multiple GFP-Rad51 foci (Figure 2B, top left panel). This correlates with exacerbation of the growth defect described above for $n m t P 3-G F P$ rad51 rrpl $\Delta$ strain cultured on YES media (Figure 1A). The opposite was seen in nmtP3-GFP-rad51 strain overexpressing $r r p 1^{+}$, where the number of cells with spontaneous GFP-Rad51 foci decreased (Figure 2B, top right panel).
The effect was subtle but significant, especially given that $r r p 1^{+}$expression is also very limited under these conditions.

Together, these results suggest that Rrpl could be involved in the control of Rad51 binding to DNA. We were interested to determine which Rrp1 activity was necessary for this regulation but because of the strong growth defect observed in the nmtP3-GFP-rad51 rrpl $\triangle$ strain, accompanied by the rapid generation and subsequent proliferation of faster-growing suppressors, complementation analysis of its phenotype was not possible, so we again employed a synthetic dosage lethality approach.

It is known that when Rad51 is overproduced in human cells, it accumulates on chromatin, forming long fibres $(35,57)$. We examined $G F P$-rad51 ${ }^{+}$overexpressing cells using fluorescence microscopy and observed that most of them contained extensive Rad51 fibres in their nuclei, often connecting several bright foci (Figure 2C). These structures virtually disappeared when Rrp1 was simultaneously overproduced, and GFP-Rad51 staining changed to diffuse with 1-3 foci (Figure 2C, D). Cells overproducing Rrp1-DAEA, an ATPase deficient mutant, contained GFP-Rad51 fibres in their nuclei (Figure 2D). This clearly demonstrates that the putative translocase activity of Rrp1 is critical in preventing excessive Rad51 accumulation on chromatin. Interestingly, even though overproduction of the Rrp1-CS mutant did supress the toxicity of GFP-rad5 $1^{+}$overexpression (Figure 1F), we were nevertheless able to detect a small, yet statistically significant, increase in the number of nuclei with Rad51 fibres in these cells (Figure 2D). This correlates with a significant increase in the number of cells with nuclear defects in the nmtP3-GFP-rad51 strain overproducing Rrp1CS as compared to wild-type Rrp1 (Figure 1F), and suggests that the putative Rrp1 ubiquitin ligase activity may also play a role in regulating Rad51 binding to DNA, although its effect is less prominent than that of the translocase activity.

When we simultaneously overproduced Rrp1-mCherry in the nmtP3-GFP-rad51 strain, GFP-Rad51 fibres were visible only in cells lacking Rrp1 signal, and about $90 \%$ of GFP-Rad51 foci co-localised with Rrp1-mCherry foci (Figure $2 \mathrm{E}$ ). We have previously shown that native Rrp1 is enriched at centromeres and $>40 \%$ of the foci it forms when overproduced are perinuclear and co-localise with Swi6 foci (14). It therefore seemed possible that Rrp1 could interact with Rad51 bound to heterochromatin regions. Under standard growth conditions Rad51 binding may occur at sites of chromosomes where replication is perturbed and this can be more frequent at sites containing repetitive sequences, such as centromeres or telomeres, which are difficult to replicate. Indeed it has been shown in $S$. pombe that Rad51 binds to centromeres in $\mathrm{S}$ phase (58).

In order to determine if Rrp1 affects Rad51 binding to centromeres we examined by chromatin immunoprecipitation the effect of $r r p 1^{+}$deletion on the ability of endogenous Rad51 to associate with outer repeat $(d g)$ and core region (cnt) of centromeres. We added other selected loci such as telomeres (tel), as another heterochromatin genomic locus, $a^{2} e^{+}$an euchromatin locus at which replication perturbation is not expected in unchallenged growth conditions (ade) and the inducible and engineered replication fork barrier (RTS1-RFB) that allows a single replisome to be blocked in 

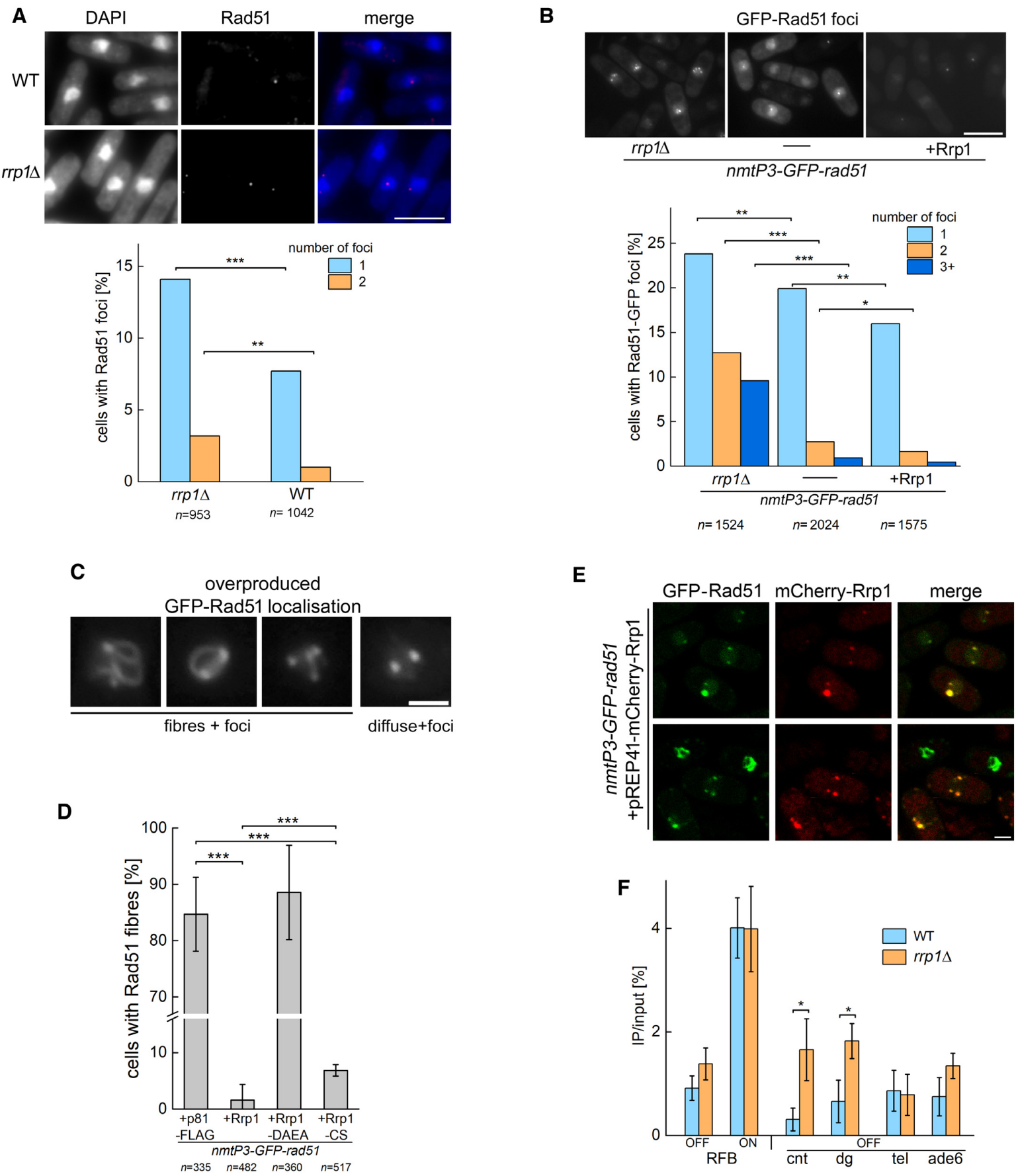

Figure 2. Rrp1 affects Rad51 localisation on chromatin. (A) $r r p 1^{+}$deletion leads to an increase in the number of spontaneous native Rad51 foci. Rad51 spontaneous foci were detected by immunofluorescence microscopy with anti-Rad51 antibodies. DAPI stained nuclei are shown as a localisation control. $\mathrm{n}=$ total number of cells examined from two independent biological replicates. Scale bar represents $10 \mu \mathrm{m}$. (B) Under conditions of mild Rad51 overproduction, deletion of $r r p 1^{+}$results in an increase in the number of cells with multiple GFP-Rad51 foci, and Rrp1 overproduction has the opposite effect. The $n m t P 3-G F P$-rad51 strain was cultured for $24 \mathrm{~h}$ on YES medium where the $n m t P 3$ promoter is mostly repressed. $n=$ total number of cells examined from two independent experiments. Scale bar represents $10 \mu \mathrm{m}$. (C, D) Two patterns of GFP-Rad51 localisation are shown: long fibres connecting discrete foci, and diffuse staining with foci. Scale bar represents $2 \mu \mathrm{m}$. In cells overexpressing both $r r p 1^{+}$and $G F P$-rad51 $1^{+}$, long Rad51 fibres are no longer observed. This effect is dependent on a functional ATPase domain, while the RING domain may play a relatively minor role. $n=$ total number of cells counted for three independent transformants of vector, $r r p 1^{+}, r r p 1-D A E A$ or rrpl-CS grown for $48 \mathrm{~h}$ in media lacking thiamine. The error bars represent the standard deviation about the mean values. The $Z$-test for two population proportions was used in all above assays to calculate the $Z$-statistic and corresponding $P$-values. (E) In cells overproducing both proteins, most GFP-Rad51 foci co-localise with mCherry-Rrp1 foci. Two panels with representative Rrp1 and Rad51 localisations are shown. Scale bar represents $2 \mu \mathrm{m}$. (F) Rrp1 influences Rad51 localisation specifically at the centromere. Analysis of Rad51 enrichment at indicated loci by ChIP-qPCR in wild-type and $r r p 1 \Delta$ strains, under conditions where a polar replication fork block was induced $(-$ thi, ON) or repressed (+thi, OFF). Primers were located at an iducible replication fork barrier (RFB), telomeres $(t e l)$, outer repeat $(d g)$ and core region (cnt) of centromeres and $a d e 6^{+}$, a random euchromatin locus (ade6). Values are mean \pm standard deviation from three independent biological replicates. Student's $t$-test was performed to calculate $P$-values. (*** $P \leq 0.001,{ }^{* *} 0.001<P \leq 0.01,{ }^{*} 0.01<P \leq 0.05$ ) 
a polar manner on chromosome III (27). The activity of the RFB is regulated by the RTS1-bound Rtf1 protein which expression is repressed in the presence of thiamine (RFB OFF) and induced in the absence of thiamine (RFB ON).

Consistent with previous reports $(59,60), \operatorname{Rad} 51$ was enriched at the active RFB (RFB ON) as compared to RFB OFF condition (Figure 2F). We observed similar level of enrichment in WT and $\operatorname{rrpl} \Delta$ strains, indicating that Rrp1 does not regulate Rad51 binding at this site-specific fork arrest. In contrast, we observed that Rad51 was significantly more enriched at both outer repeat $(d g)$ and core region (cnt) of centromeres in rrpl $\Delta$ than in $W T$ cells $(P$ values 0.036 and 0.04 for $d g$ and $c n t$, respectively) whereas no higher enrichment was observed at telomeres and $a^{d e} \sigma^{+}$ locus ( $P$-values 0.85 and 0.13 for $t e l$ and ade6, respectively) (Figure 2F).

This result supports the notion that Rrp1 regulates Rad51 bound to specific sites or DNA structures, such as those present within centromeric regions. This agrees with the previously proposed role for Rrp1 in maintaining centromere stability (14), although further studies are needed to examine how these Rrp1 activities are connected.

\section{The role of Rrp1 in regulating Rad51-induced toxicity is in- dependent from Rrp2}

Overproduction of Rrp2 in a rad51 $1^{+}$overexpressing strain was unable to suppress the growth defect (Figure 1C, D), viability loss (Supplementary Figure S1A, C) and the chromosome segregation defect (Supplementary Figure S1F), and did not prevent the accumulation of Rad51 fibres on chromatin (Supplementary Figure S1G). Moreover, rescue of the GFP-rad51 $1^{+}$overexpression-induced growth defect by overproduction of Rrpl was not affected by the presence of Rrp2 (Supplementary Figure S1H) or the recombination auxiliary factor complex Swi5-Sfr1 (Supplementary Figure S1I). This suggests that the observed role of Rrpl in regulating $\operatorname{Rad} 51$ is distinct from the previously described mutually dependent role of Rrp1 and Rrp2 in the Swi5-Sfr1 sub-pathway of HR $(12,13)$.

\section{Purified Rrp1 binds to DNA and has a DNA-dependent AT- Pase activity}

In order to gain mechanistic insight into the function of Rrp1, recombinant Rrp1-FLAG was purified to nearhomogeneity following overexpression in Escherichia coli (Supplementary Figure S2A).

Since Rrp1 was predicted to have ATPase activity (61), we first examined if the purified protein could indeed hydrolyse ATP. In the absence of DNA, very low ATPase activity was detected. However, robust ATP hydrolysis was observed in the presence of dsDNA and a lower ATPase activity was seen in the presence of short oligo ssDNA (Supplementary Figure S2B). These results indicate that Rrp1 has an ATPase activity preferentially stimulated by dsDNA. In order to confirm that the observed ATPase activity is not derived from contaminating protein(s) in the Rrpl protein preparation, we measured the ATPase activity and protein concentration in the peak fractions from the final purification step. The dsDNA-dependent and -independent ATPase activities corresponded with protein concentration signal for
Rrp1, indicating that the observed ATPase activities are intrinsic properties of the Rrp1 protein (Supplementary Figure S2C).

The observed dependency on DNA for ATP hydrolysis suggested that Rrpl is capable of binding DNA. This was tested by electrophoretic mobility-shift assays (EMSA). At concentrations as low as $0.05 \mu \mathrm{M}$ (Rrp1: nucleotide ratio of 1:100), all circular ssDNA (cssDNA) was shifted in an ATP-independent manner by Rrp1, and this shift was enhanced at higher concentrations of protein (Supplementary Figure S2D). A lesser shift was observed with linearized dsDNA (ldsDNA), with some unbound DNA remaining even at $0.30 \mu \mathrm{M}$ Rrp1 (Rrp1: base pair ratio of 1:8.33), although ATP was also dispensable for this binding (Supplementary Figure S2E). Some signal was observed in the wells, particularly for dsDNA, suggesting that Rrp1 may form aggregates consisting of protein-DNA networks that are too large to enter the gel. Rrp1 binding to dsDNA lacking free DNA ends (covalently closed circular DNA, cccDNA) was comparable to cssDNA, with the exception that no shift was observed at $0.05 \mu \mathrm{M}$ of Rrp1 (Supplementary Figure S2F). Taken together, these results indicate that Rrp1 binds both ssDNA and dsDNA in an ATP-independent manner, with slightly higher affinity for ssDNA.

\section{Rrp1 physically interacts with Rad51}

The genetic interactions observed between Rad51 and Rrp1 raised the possibility that the two proteins interact physically. To investigate this possibility, we first employed the yeast two-hybrid system (Y2H). For Rad51, two constructs were used: a short N-terminal fragment $(\operatorname{Rad} 51-N)$, and a long C-terminal fragment containing the Walker $\mathrm{A}$ and B motifs (Rad51-C) (Figure 3A). We observed a robust growth of transformants containing genes for Rrp1 and Rad51-C on high stringency SD DO-4 plates, indicating that the site of putative Rrp1 binding lies within the Rad51 region containing Walker $\mathrm{A}$ and $\mathrm{B}$ motifs (Figure $3 \mathrm{~B}$ ). In order to map the corresponding region within Rrpl that is responsible for Rad51 binding, we created a series of four truncated forms of Rrp1 (Figure 3C) and repeated the $\mathrm{Y} 2 \mathrm{H}$ assay with Rad51-C. These experiments revealed that the fragment of Rrp1 containing its C-terminal helicase domain was sufficient for the interaction with Rad51 (Figure 3D). In agreement with our genetic data, no interaction was observed between Rad51 and Rrp2 by $\mathrm{Y} 2 \mathrm{H}$ (Figure 3B).

To validate these $\mathrm{Y} 2 \mathrm{H}$ results and rule-out the possibility that the observed Rrp1-Rad51 interaction involved an intermediary molecule, purified Rad51 and purified Rrp1FLAG were mixed together and subjected to immunoprecipitation with anti-FLAG M2 agarose. Rad51 was seen to co-immunoprecipitate with Rrp1-FLAG (Figure 3E), thus confirming the existence of a direct interaction between these two proteins. Furthermore, by immunoprecipitating endogenously expressed Rad51 with overproduced Rrp1FLAG from native protein extracts, we were able to demonstrate that the Rad51-Rrp1 complex is formed in vivo in $S$. pombe cells (Figure 3F). We did not see such complex formation between Rad51 and Rrp2-FLAG. 
A
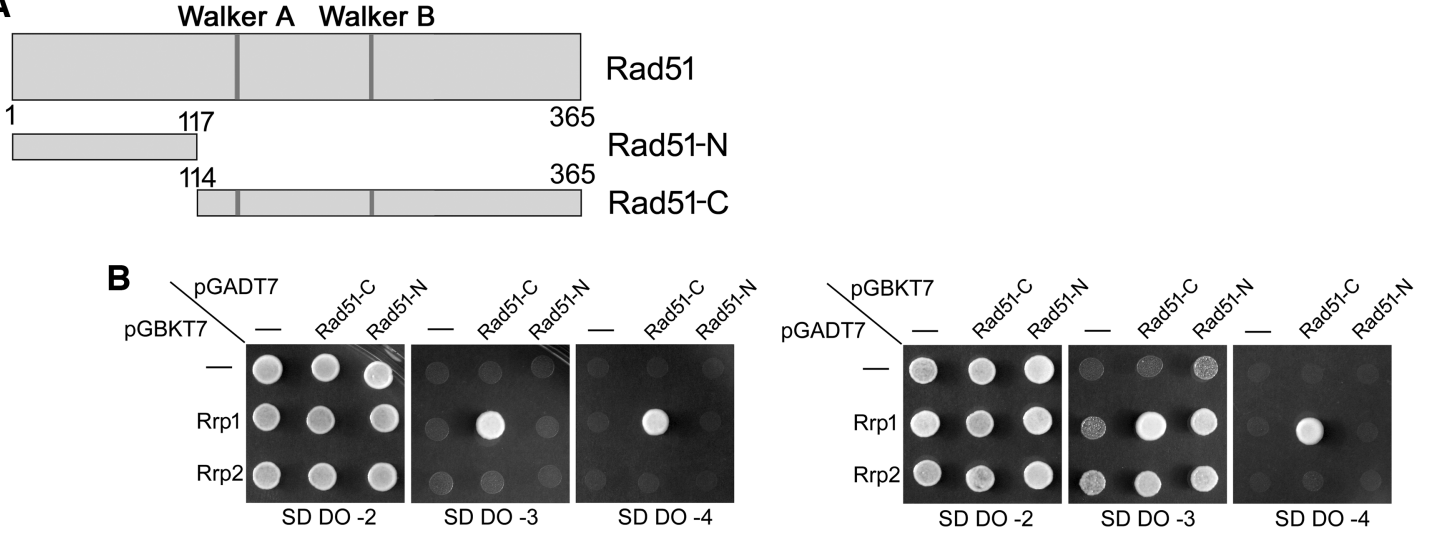

C
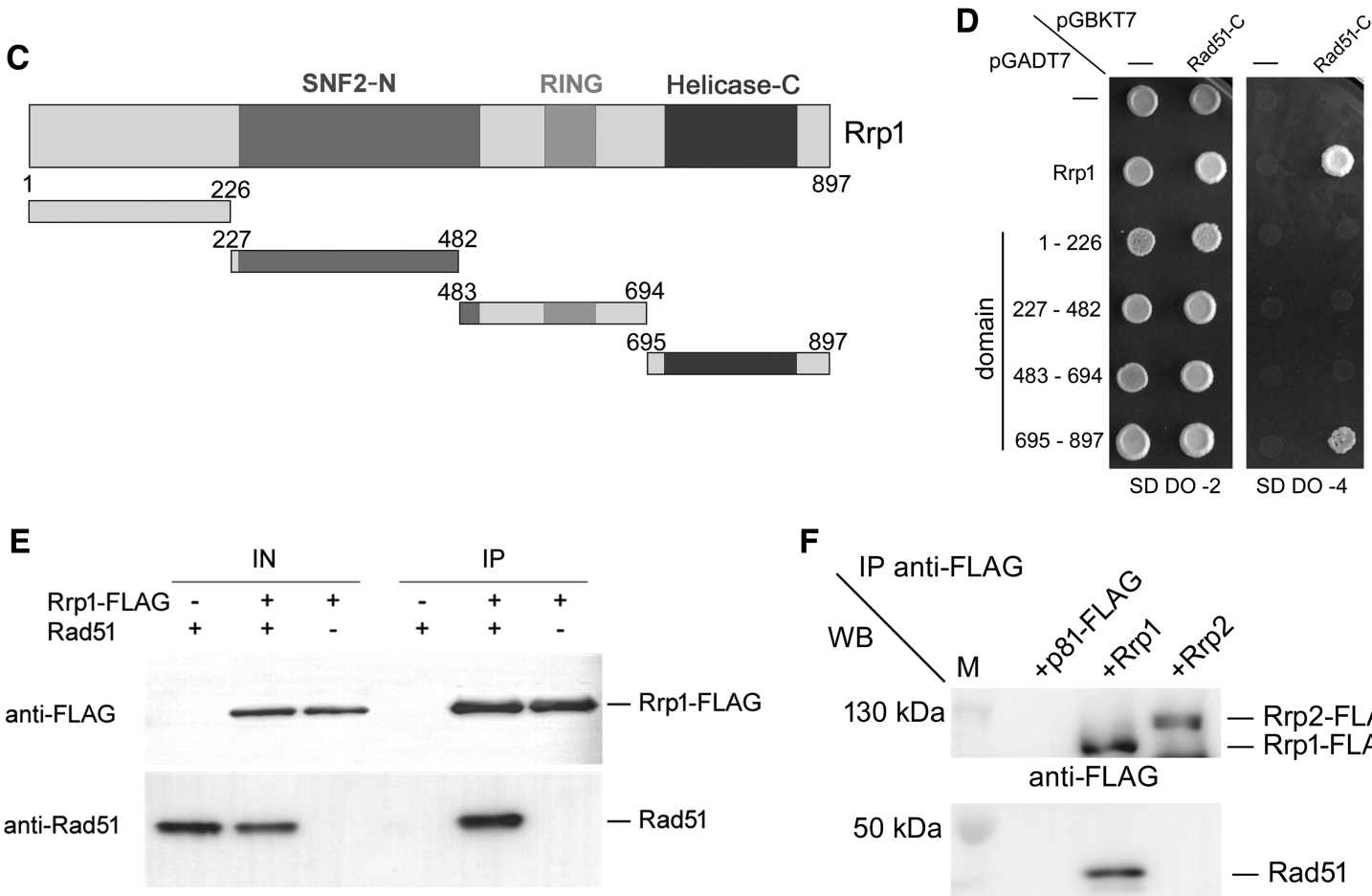

$\mathbf{F}$
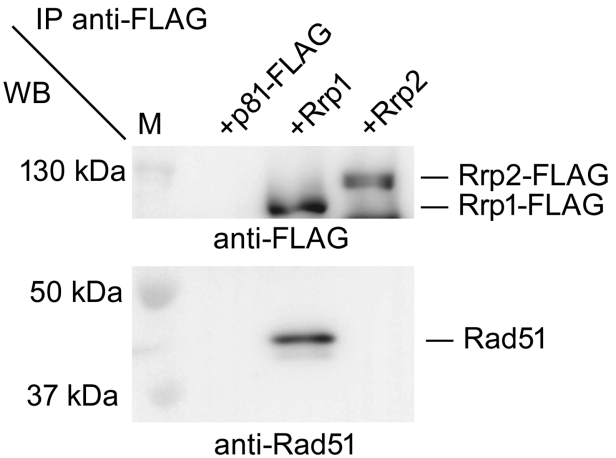

Figure 3. Rrp1 directly interacts with Rad51. Analysis of the Rrp1 interaction with Rad51 by yeast two-hybrid analysis. (A) Two Rad51 constructs were used: Rad51-N (N-terminal, residues 1-117) and Rad51-C (C-terminal, residues 114-365 containing the Walker A and B motifs). (B) Rad51-C (pGBKT7 and pGADT7 plasmid) is involved in the interaction with Rrp1 (self-activation is observed in the transformant with pGADT7-Rrp1 and empty pGBKT7 vector, right panels, suggestive of Rrpl DNA binding activity). Transformants were selected on synthetic dextrose drop-out medium without Leu and Trp (SD DO-2), then plated on low stringency medium without Leu, Trp and His (SD DO-3) and high stringency medium without Leu, Trp, His and Ade (SD DO-4). (C) Schematic representation of a series of four truncated forms of Rrp1 used to map the site of interaction with Rad51. (D) The Cterminal fragment of Rrp1 (residues 695-897) containing the C-helicase domain was found to interact with Rad51, determined as described for (B). (E) Rad51 immunoprecipitated with Rrp1-FLAG in vitro. Purified Rad51 and purified Rrp1-FLAG were mixed and incubated with anti-FLAG M2 agarose. Proteins were eluted from the beads with 3xFLAG peptide and separated by SDS-PAGE, then analysed by Western with anti-FLAG antibody and antiRad51 antiserum. -, protein was omitted and the equivalent volume of protein storage buffer was added instead. + , protein was included. The experiment was repeated twice. (F) Rad51 interacts with Rrp1-FLAG, but not with Rrp2-FLAG, in vivo. Protein extracts prepared from cells overexpressing either rrp1-FLAG (three independent transformants were examined) or $r r p 2-F L A G$, or transformed with empty vector as a control (pREP81-FLAG plasmid), were incubated with anti-FLAG M2 agarose. Proteins were then eluted with 3xFLAG peptide, separated by SDS-PAGE, and analysed by Western with anti-FLAG antibody and anti-Rad51 antiserum. 


\section{Rrp1 dissociates Rad51 from dsDNA in vitro}

SWI2/SNF2-related translocases have been proposed to remove Rad51 from dsDNA in heteroduplex DNA and deadend non-productive complexes, both in yeast and human cells $(35,39)$. The existence of a physical interaction between Rrp1 and Rad51, combined with the ability of Rrp1 to suppress erroneous association of Rad51 with chromatin (Figures 2 and 3), prompted us to examine whether Rrpl can directly counteract Rad51 binding to linearized dsDNA. To test this, we exploited the fact that the binding of purified Rrp1 to dsDNA results in a distinctive EMSA pattern where most of the dsDNA signal is retained in the well (Supplementary Figure S2E). This pattern is easily distinguishable from the binding of Rad51 to DNA, which produces a smear at low concentrations of protein $(0.5 \mu \mathrm{M})$ and a discrete band at higher concentrations (1.5 or $3 \mu \mathrm{M}$; Figure 4A). dsDNA was first coated with different concentrations of Rad51 and then challenged with sub-stoichiometric amounts of Rrp1. Protein-DNA complexes were then resolved by gel agarose electrophoresis. Compared to the condition where Rrp1 was omitted, the bands for Rad51bound DNA became fainter when $0.1 \mu \mathrm{M}$ of Rrp1 was included. Moreover, the inclusion of $0.3 \mu \mathrm{M}$ of Rrpl led to a drastic decline in the signal of Rad51-dsDNA bands, even when the dsDNA was precoated with five-fold more Rad51 molecules, and signal in the well became apparent. These results suggest that sub-stoichiometric amounts of Rrpl effectively outcompete Rad51 for binding to dsDNA. Since Rrpl can bind to both dsDNA (Supplementary Figure S2E, F) and $\operatorname{Rad} 51$ (Figure 3E), an alternative explanation for these results is that, rather than displace Rad51 from dsDNA, Rrp1 binds to Rad51-dsDNA complexes, leading to the formation of higher-order complexes that are unable to enter the gel.

It has been shown before that Rad51 protein strongly binds to dsDNA and its turnover is low both in yeast and humans $(62,63)$. Nevertheless, the addition of scavenger DNA helps to visualise dissociation of Rad51 molecules (64). Thus, in order to more directly examine if Rrp1 could remove Rad51 from dsDNA, we analysed dissociation of Rad51 from fluorescently labeled dsDNA by measuring fluorescence anisotropy in real-time in the presence of a vast excess of scavenger ssDNA (Figure 4B). In this assay, Rad51 was first bound to fluorescently-labeled dsDNA to form a filament. This induced a decrease in the mobility of dsDNA, observed as an increase in fluorescence anisotropy ( $\Delta$ anisotropy value of approximately 0.1 ). Even after the addition of scavenger ssDNA, the $\Delta$ anisotropy values decreased only slightly over 500s (black line in Figure 4C), indicating that the Rad51-dsDNA filament is relatively stable, consistent with reports described above. Upon Rrpl addition to the reaction mixture containing the Rad51-dsDNA filament and scavenger ssDNA, the $\Delta$ anisotropy values decreased rapidly, indicating that Rad51 was being cleared from dsDNA (purple line in Figure 4C). A fit with $\propto \exp (-$ $k_{\text {off }} \cdot t$ ) function gave a good approximation to our experimental data and yielded $k_{\text {off }}$ rate, corresponding to dissociation rate, for $\operatorname{Rad} 51, k_{\mathrm{off}}=1.63 \times 10^{-3} \mathrm{~s}^{-1}$, (fit quality, $\left.R^{2}=0.948\right)$ and for the removal of $\operatorname{Rad} 51$ by $\operatorname{Rrp} 1, k_{\text {off }}=$ $(9.46 \pm 0.53) \times 10^{-3} \mathrm{~s}^{-1}$, (fit quality, $\left.R^{2}>0.994\right)$. This al- lowed us to estimate that in our experimental setup Rad51 dissociates from dsDNA almost six-fold faster in the presence of Rrp1. While further extensive, in-depth analysis is required to determine the influence of Rrp1 on Rad51 dissociation kinetics, our data directly demonstrate that Rrp1 is involved in the removal Rad51 from dsDNA.

A control experiment showed that when the same amount of Rrp1 was added to the dsDNA alone in the absence of Rad51, it elicited a slight increase in anisotropy (blue line in Figure 4C), thus ruling out the possibility that Rrp1 modifies the dsDNA in some way that reduces anisotropy.

We also examined the effect of Rrp1 on Rad51-ssDNA complexes. Although a slight decrease in the intensity of Rad51-ssDNA bands was observed by EMSA (Supplementary Figure S3A), this was negligible when compared with the effect of Rrp1 on Rad51-dsDNA complexes (Figure 4A). Consistently, relative anisotropy increased upon addition of Rrp1 to Rad51-ssDNA complexes in a concentration-dependent manner (Supplementary Figure S3B), suggesting that rather than dissembling Rad51-ssDNA complexes, Rrp1 was binding to them. This binding to Rad51-ssDNA filaments may lead to modulation of their activity, but further studies are needed to conclusively test this hypothesis.

\section{Rrp1 has an E3 ubiquitin ligase activity with Rad51 as a sub- strate}

In addition to an ATPase domain, Rrp1 also has a Zinc finger RING-type domain characteristic of E3 ubiquitin ligases (61), and belongs, together with its $S$. cerevisiae orthologue U1s1, to the RING-domain-containing Rad5/16like group of SWI2/SNF2 translocases, distinct from the RAD54 family $(65,66)$.

We therefore hypothesized that Rrp1 may have an E3 ligase activity with Rad51 as a substrate. In order to directly test this possibility, in vitro $\operatorname{Rad} 51$ ubiquitylation assays were performed using ubiquitin, Ubal (E1) and Ubc4 (E2) enzymes purified from E. coli, and purified Rrp1FLAG as the sole E3 ubiquitin ligase enzyme. Reaction products were separated by SDS-PAGE and subjected to western blot analysis. Multiple high molecular weight protein bands were observed only when all assay components were included in the reaction (Figure 5A). Strikingly, a similar banding pattern was detected with both anti-Rad51 and anti-Ubiquitin antibodies, indicating that these bands represent ubiquitylated forms of Rad51. Consistent with this notion, the unmodified Rad51 band decreased in intensity only in the assay with all reaction components (Figure 5A). To further validate these findings, we repeated this ubiquitylation assay with either wild-type Rrp1 protein or the Rrp1CS variant, where the Rrp1 RING domain was inactivated, as the sole E3 ligase. When Rrp1-CS was employed, the characteristic protein ladder was not formed (Figure 5B), indicating that Rrp1 E3 ubiquitin ligase activity was responsible for Rad51 ubiquitylation.

Interestingly, when the membrane was probed with an anti-FLAG antibody to detect Rrp1 protein, several high molecular weight protein bands greater in mass than Rrp1 were observed in reactions containing wild-type Rrp1 but not Rrp1-CS (Figure 5B, bottom panel), indicating that 
A

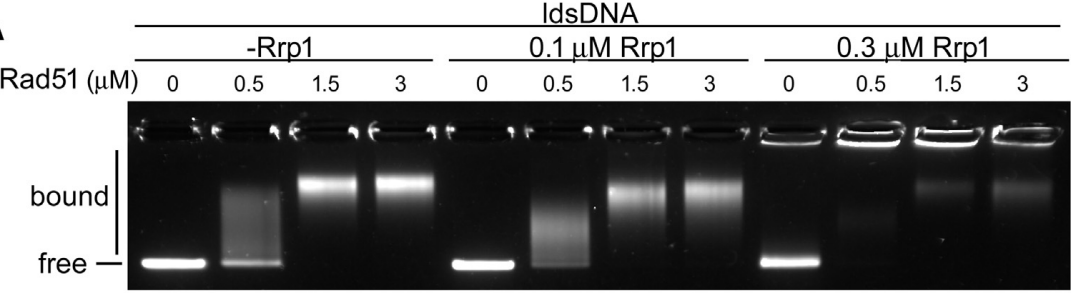

B

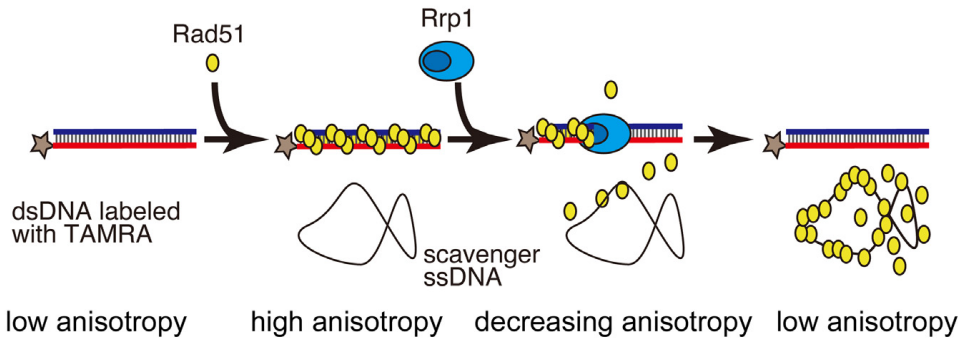

C

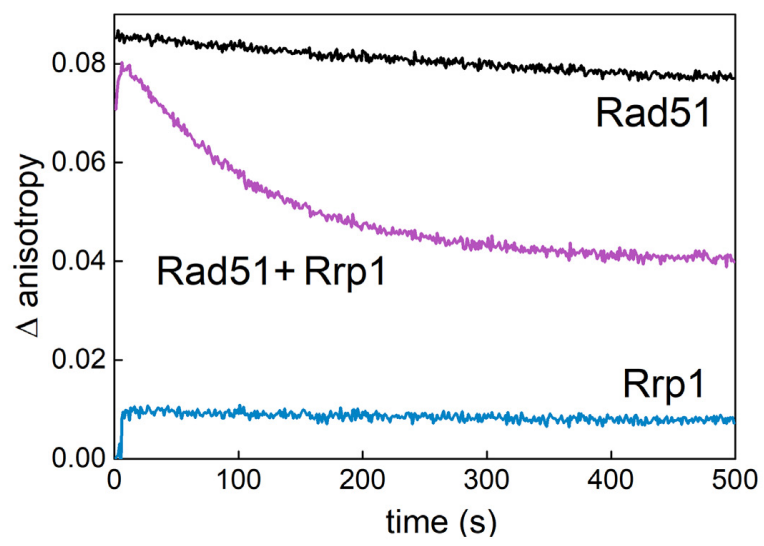

Figure 4. Rrp1 can disassemble Rad51-dsDNA complexes. (A) Rrp1 outcompetes Rad51 for binding to dsDNA as demonstrated by electrophoretic mobility shift assay (EMSA). Increasing amounts of Rad51 were pre-incubated with linear double-stranded DNA (ldsDNA) before addition of the indicated concentration of Rrp1. Mixtures were resolved on an agarose gel and stained with SYBR-gold. (B) Diagram of the fluorescence anisotropy assay used to measure in real-time the dissociation of Rad51 from fluorescently labelled dsDNA. Rad51 $(6 \mu \mathrm{M})$ was incubated with a dsDNA oligonucleotide $(3 \mu \mathrm{M}$ base pair concentration) labelled with the TAMRA fluorophore. Unlabelled heterologous scavenger ssDNA was then added, followed by a sub-stoichiometric amount of $\operatorname{Rrp} 1(0.25 \mu \mathrm{M})$ or the equivalent volume of protein storage buffer, and fluorescence anisotropy was monitored. (C) Time course of Rad51 disassembly from dsDNA following the addition of Rrpl was performed for two independent Rrp1 preparations. A representative result is shown, with the comparative decline in anisotropy ( $\Delta$ anisotropy) observed in the three independent reactions containing Rrp1, indicating that Rad51-dsDNA complexes are disassembled.

Rrp1 is capable of auto-ubiquitylation. Consistent with this notion, the intensity of the unmodified Rrp1 band was decreased in these reactions.

When the Rad51 ubiquitylation assay was performed with $\operatorname{Rad} 51$ preassembled on dsDNA, the intensity of bands corresponding to ubiquitylated Rad51 decreased in a dose dependent manner with increasing concentrations of DNA (Figure 5C). This was especially evident for polyubiquitylated $\operatorname{Rad} 51$ species, and demonstrates that $\operatorname{Rad} 51$ ubiquitylation by Rrp1 is inhibited by dsDNA. The precise mechanism however, whereby dsDNA inhibits this ubiquitylation, remains to be elucidated.

In order to determine if Rrp1-dependent ubiquitylation of endogenous Rad51 can be detected in vivo, we prepared protein extracts from cells expressing His $_{6}$-tagged Ubiquitin from a strong $n m t$ promoter $(n m t l)$ and overproduc- ing Rrp1. Precipitates from the His pull-down fractions were analysed by Western blot and probed with anti-Rad51 antibody. We observed an accumulation of high molecular weight Rad51 species, and these were substantially less abundant in cells overproducing the Rrp1-CS mutant protein, or carrying an empty vector (Figure 5D). This bolsters our in vitro observations (Figure 5A, B) that Rrp1 has the ability to ubiquitylate Rad51 in a manner dependent on its functional RING domain.

Polyubiquitylation is a well-established signal for protein degradation (67). We did not observe by Western blot any significant changes in native Rad51 protein levels in total extract fractions upon $r r p I^{+}$deletion or overexpression (Supplementary Figure S4A, upper-right graph). However, after prolonged overexpression of rrp1-CS, Rad51 accumulation could be detected (Supplementary Figure S4A 
A

$\begin{array}{rcccccc}\text { Rad51 } & - & + & + & + & + & + \\ \text { Rrp1-FLAG E3 } & + & + & - & + & + & + \\ \text { Ubc4 E2 } & + & + & + & - & + & + \\ \text { Uba1 E1 } & + & + & + & + & - & + \\ \text { Ubiquitin } & + & + & + & + & + & -\end{array}$
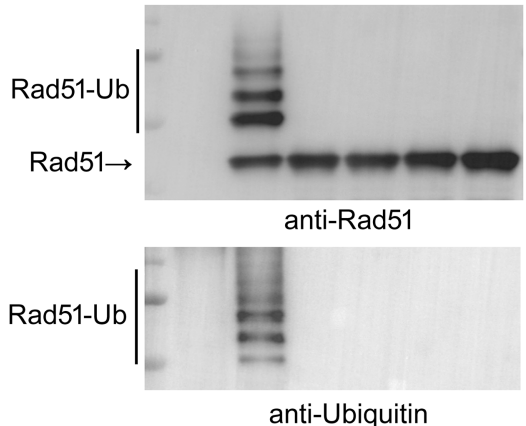

anti-Ubiquitin

C

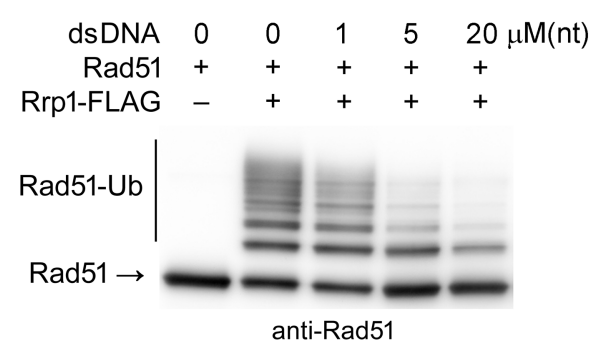

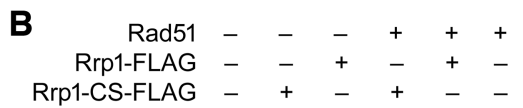

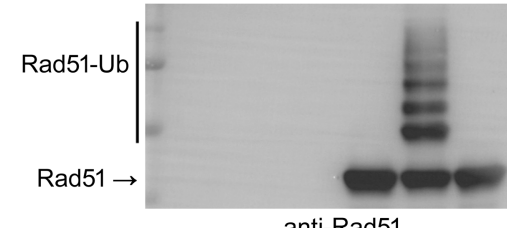

anti-Rad51

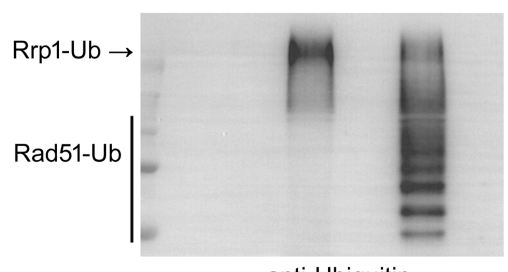

anti-Ubiquitin

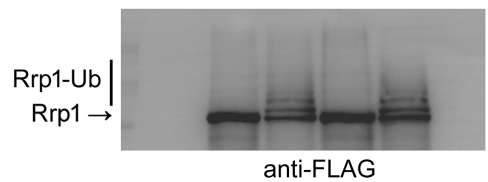

D WCE His pull-down

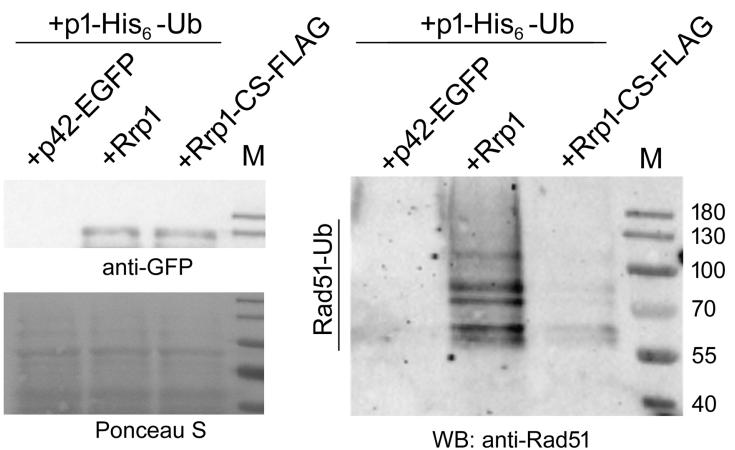

Figure 5. Rrp1 is an E3 ubiquitin ligase with Rad51 as a substrate. (A) Rrp1 ubiquitylates Rad51 in vitro. The indicated reaction components were included (+) or omitted (-) for in vitro ubiquitylation assays. After the reaction, the reaction mixture was analysed by western blotting with anti-Rad51 antiserum and anti-Ubiquitin antibodies, and multiple bands indicative of Rad51 ubiquitylation (Rad51-Ub) are shown. (B) Rrp1 RING domain is critical for Rad51 ubiquitylation. In vitro ubiquitylation assay containing all components as in (A) with Rrp1-FLAG or Rrp1-CS-FLAG as the E3 ligase. The reaction mixture was analysed by western blotting with anti-Rad51 antiserum and anti-Ubiquitin antibodies. Additionally, reaction products were analysed with anti-FLAG antibodies (lowest panel), revealing auto-ubiquitylation of Rrp1 (Rrp1-Ub). (C) The presence of DNA limits ubiquitylation of Rad51 by Rrp1. In vitro ubiquitylation assay containing all components as in (A) with pre-formed Rad51-dsDNA complexes obtained by incubation of $1 \mu \mathrm{M}$ Rad51 with indicated concentrations of dsDNA. The reaction mixture was analysed by western blotting with anti-Rad51 antiserum. (D) Rrp1 overproduction leads to the accumulation of ubiquitylated forms of Rad51 in vivo. The wild-type strain (WT) was co-transformed with a pREP1-based plasmid encoding hexahistidine-tagged Ubiquitin (+p1-His $6-\mathrm{Ub})$ together with pREP42 based plasmid (p42-EGFP) harbouring genes for Rrp1 or Rrp1CS-FLAG. After $24 \mathrm{~h}$ growth under expression inducing conditions, ubiquitylated proteins were isolated by His pull-down and detected by western blot with Rad51 antiserum. Experiment was repeated twice for independent sets of transformants. Rrp1 and Rrp1-CS protein levels were comparable, as seen in whole cell extracts (WCE) probed with anti-GFP antibodies.

bottom-left graph). Rad51 levels increased both in nucleoplasm and chromatin fractions obtained from cells overexpressing rrpl-CS, (Supplementary Figure S4B), suggesting that Rrp1 may have a role in Rad51 turnover that is dependent on its ubiquitin ligase activity.

This result is somewhat surprising since overproduction of wild-type Rrp1 does not lead to a reduction in Rad51 levels that is detectable by Western blotting. We propose that under standard growth conditions Rrp1 modulates the activity of only a small fraction of Rad51 molecules, as was shown by Rad51-ChIP (Figure 2F), meaning that global
Rad51 turnover is virtually unaffected by Rrp1 overproduction. Only when degradation of this subset of Rad51 molecules, presumably removed from DNA by Rrp1, is defective, as in the case of prolonged overexpression of rrp1CS, limited Rad51 accumulation can be detected by Western blotting.

In this work we have demonstrated that high levels of Rad51 are toxic to $S$. pombe cells and, as discussed above, rrp1-CS overexpression results in the increase in Rad51 levels. Yet, as shown previously (14) rrpl-CS overexpression does not lead to a growth defect. We reason that other ubiq- 
uitin ligases might be involved in removing excess Rad51 from cells overproducing Rrp1-CS mutant, so Rad51 levels on DNA do not rise above the threshold that would lead to growth inhibition. Indeed, in the $f b h l \Delta$ strain, which is devoid of a component of an E3 complex that can ubiquitylate Rad51 (44), overexpression of rrpl-CS is more toxic than overexpression of wild-type rrpl+ (Supplementary Figure S4C).

We thus identify Rrp1 as an ATPase and translocase that can remove Rad51 from dsDNA, and as a ubiquitin ligase with Rad51 as its substrate. We propose that these Rrp1 activities are important for the regulation of Rad51 function.

\section{DISCUSSION}

Previous studies have shown that the presence of the RAD54 family of SWI2/SNF2 DNA translocases, RAD54L and RAD54B in humans (35), and Rdh54 and Rad54 in S. cerevisiae (39), is necessary to counteract the genotoxic effects of Rad51 overproduction. Another budding yeast protein Uls1, has also been shown to participate in modulating Rad51 activity. S. pombe Rrp1 and Rrp2 are Uls1 orthologues and all three proteins belong to a unique Rad5/16-like group of SWI2/SNF2 DNA translocases, that contain both an ATPase domain and a Zinc finger RING-type domain characteristic of E3 ubiquitin ligases $(65,66)$.

In this work, we demonstrated that toxicity of $\mathrm{rad} 51^{+}$ overexpression was increased in a $\operatorname{rrpl} \Delta$ but not in a $\operatorname{rrp} 2 \Delta$ strain. This implied that, in $S$. pombe, Rrp1 has a prominent function, independent from Rrp2, in counteracting the toxicity of Rad51 overproduction. This is in contrast to the requirement for Uls1 in $S$. cerevisiae, which only becomes apparent in the absence of both Rdh54 and Rad54 (39). We thus examined the contributions of putative translocase and ubiquitin ligase activities of Rrp1 to its interaction with Rad51.

We found that counteracting the cellular toxicity of Rad51 overexpression requires the ATPase but not the RING domain. Similarly, the deleterious consequences of Rad51 overexpression on chromosome segregation are rescued via the ATPase domain of Rrp1, suggesting that Rrp1 modulates Rad51 through its translocase activity. Interestingly, however, we found that the Rrp1-CS mutant, with an inactivated RING domain, was slightly less proficient in counteracting inappropriate Rad51 accumulation on chromatin and the appearance of aberrant DNA segregation events. This raised an interesting possibility, not examined for its orthologue Uls1, that Rrp1 ubiquitin ligase activity may play a role in Rad51 regulation, even though it was apparently not crucial for suppression of the Rad51 overproduction-induced growth defect.

Importantly, Rrp1 is also involved in regulating Rad51 when the latter is not overproduced. We found that the number of spontaneous Rad51 foci under endogenous expression conditions increased in cells lacking Rrpl. Moreover, Rad51-ChIP demonstrated that Rrp1 has no global effect on Rad51 binding, and does not influence its levels even at blocked replication forks, where Rad51 is significantly enriched. Instead, Rrp1 specifically affects the ability of endogenous Rad51 to associate with centromeres. This sug- gests that the physiological role of Rrp1 may be to regulate a subpopulation of Rad51 molecules bound to specific sites or DNA structures, such as those present within centromeric regions. It has been previously shown that $S$. pombe Rad51 localises to centromeres (58), and together with another SWI2/SNF2 translocase, Rad54, promotes recombination between centromere repeats that is important for chromosome stability (68). This indicates that two SWI2/SNF2 translocases, Rad54 and Rrp1, may participate in Rad51 regulation that is important for the maintenance of centromere function.

Purification of Rrp1 allowed us to obtain mechanistic insight into its function and biochemical activities. Purified Rrp1 binds to both ssDNA and dsDNA independently of ATP, and has a DNA-dependent ATPase activity. Importantly, we demonstrated that Rrp1 and Rad51 interact both in vitro and in vivo, supporting our conclusion that Rad51 may be the direct target of Rrp1 activity. Indeed, using an in vitro fluorescence anisotropy assay, we showed that Rrp1 can efficiently dissociate Rad51 from dsDNA, establishing Rrp1 as a translocase that counteracts Rad51 binding to chromatin to limit its genotoxicity. Our data thus indicate that the Rad5/16-like group of SWI2/SNF2 translocases also participates in limiting Rad51-mediated toxicity, as shown for RAD54-like DNA translocases (35).

Our work suggests that the RING domain of Rrp1 may also be involved in Rad51 regulation. We provide direct evidence that Rad51 undergoes polyubiquitylation that is dependent on the RING domain of Rrp1, in vivo and in vitro. This indicates that Rrp1 has an E3 ubiquitin ligase activity and Rad51 is one of its substrates.

Because the RING domain is mostly dispensable to counteract the toxicity of Rad51 overexpression, we propose that Rad51 ubiquitylation by Rrp1 is not an absolute prerequisite for Rad51 removal from DNA by Rrp1's translocase activity. Nevertheless, examination of $\mathrm{rad} 5 \mathrm{I}^{+}$-overexpressing cells simultaneously overproducing Rrp1-CS or wild-type Rrp1 revealed that the number of cells with Rad51 fibres and segregation defects was increased in the former, implying that inactivation of the RING domain does affect the ability of Rrp1 to prevent Rad51 association with DNA.

Rad51 ubiquitylation has previously been shown to compromise its ability to bind DNA $(43,45)$ and it has been proposed that the FBH1 translocase displaces RAD51 from ssDNA and ubiquitylates it to prevent its reloading (45). Since the efficiency of Rrpl-mediated ubiquitylation of Rad51 preassembled on dsDNA was markedly decreased, we speculate that a similar two-step model could be applicable for Rrp1: Rrp1 translocase could displace Rad51 from dsDNA and ubiquitylate it to prevent its reloading. However, since we do not know how DNA inhibits Rad51 ubiquitylation, more studies are needed to understand how the Rrp1 ATPase/translocase and ubiquitin ligase activities cooperate in counteracting excessive Rad51 binding to chromatin.

Rad51 polyubiquitylation by RFWD3 not only inhibits its DNA binding but also leads to its proteasomal degradation (43). In accord with our assumption that Rrp1, which is an extremely low abundance protein, interacts only with a small fraction of Rad51 molecules and does not affect global Rad51 turnover, we did not detect any significant 
changes in native Rad51 protein levels upon $r r p 1^{+}$deletion or overexpression. However, Rad51 levels increased in cells overexpressing rrpl-CS, suggesting that Rrp1 ubiquitin ligase activity may have a role in promoting the proteolytic degradation of a specific subset of Rad51 molecules.

Members of the Rad5/16-like group of SWI2/SNF2 translocases, such as budding yeast $\operatorname{Rad} 5$ and human SHPRH and HLTF, have been shown thus far to ubiquitylate PCNA (69). Recently, SHPRH has been identified as a nucleosome E3-ubiquitin-ligase (70), and we have demonstrated that Rrp1 is involved in modulation of nucleosome dynamics important for centromere, and thus genome, stability (14). Our present work, describing the Rrp1-Rad51 interaction, which involves ATPase/translocase and ubiquitin ligase activities of Rrp1, extends the possible range of functions performed by this class of SNF2 enzymes and may contribute to a better understanding of their role in modulating specific activities at perturbed replication forks and at regions of the genome that are difficult to replicate, such as centromeres.

Rad51 is overproduced in several types of human cancers $(71,72)$ and multiple cancer cell lines $(73,74)$, and contributes to their increased survival after DSB induction. Increased levels of Rad51 may compensate for deficiencies in other DNA repair pathways in cancer cells and are often associated with poor patient survival prognosis $(75,76)$. Since the role of ubiquitylation has not been addressed in previous studies on Rad51 dysregulation by Swi2/Snf2-related translocases $(35,39)$, our work may have implications for human health.

\section{DATA AVAILABILITY}

Source data as well as strains and plasmids are available upon request.

\section{SUPPLEMENTARY DATA}

Supplementary Data are available at NAR Online.

\section{ACKNOWLEDGEMENTS}

We thank Anna Barg-Wojas and Kornel Milcarz for their involvement in the initial stages of this project, Piotr Wiśniewski for help with data presentation and stimulating discussions, and Antony M. Carr for encouraging comments on the manuscript. The funders had no role in designing the study, data collection and analysis, decision to publish, or preparation of the manuscript.

Author contributions: Jakub Muraszko, Formal analysis, Investigation, Methodology, Funding acquisition, Writing original draft; Karol Kramarz, Formal analysis, Investigation, Methodology, Funding acquisition, Writing - review and editing; Bilge Argunhan, Formal analysis, Methodology, Resources, Funding acquisition, Writing - review and editing; Kentaro Ito, Formal analysis, Investigation, Methodology; Hideo Tsubotuchi, Investigation, Funding acquisition, Writing - editing; Gabriela Baranowska, Investigation, Funding acquisition; Yumiko Kurokawa, Investigation, Methodology, Resources; Yasuto Murayama,
Methodology, Supervision; Sarah Lambert, Funding acquisition, Writing - review and editing; Hiroshi Iwasaki, Supervision, Funding acquisition, Methodology, Writing - review and editing; Dorota Dziadkowiec, Conceptualization, Supervision, Formal analysis, Funding acquisition, Investigation, Methodology, Writing - original draft, review and editing.

\section{FUNDING}

National Science Centre, Poland: Harmonia 5 [2013/10/M/NZ1/00254 to D.D.], Preludium 11 [2016/21/N/NZ1/02828 to J.M.], in part by Preludium 13 [2017/25/N/NZ1/01974 to G.B.]; Grants-in-Aid for Scientific Research [15H059749 to H.I., 18H02371 to H.T.]; Young Scientists [17K15061 to B.A.]; Early-Career Scientists [20K15713 to B.A.] from the Japan Society for the Promotion of Science (JSPS); Ligue contre le cancer 'Equipe Labellisée 2020 [EL2020LNCC/Sal]; ANR grant NIRO [ANR-19-CE12-0023-01]; Fondation ARC [PJA 20181208114 to S.L.]; Fondation ARC [PDF 20171206749 to K.K.]. Funding for open access charge: the Excellence Initiative - Research University (IDUB) programme for the University of Wroclaw.

Conflict of interest statement. None declared.

\section{REFERENCES}

1. Kolinjivadi,A.M., Sannino,V., de Antoni,A., Técher,H., Baldi,G. and Costanzo,V. (2017) Moonlighting at replication forks - a new life for homologous recombination proteins BRCA1, BRCA2 and RAD51. FEBS Lett., 591, 1083-1100.

2. Symington,L.S. (2002) Role of RAD52 epistasis group genes in homologous recombination and double-strand break repair. Microbiol. Mol. Biol. Rev., 66, 630-670.

3. Kowalczykowski,S.C. (2015) An overview of the molecular mechanisms of recombinational DNA repair. Cold Spring Harb. Perspect. Biol., 7, a016410.

4. Krogh,B.O. and Symington,L.S. (2004) Recombination proteins in yeast. Annu. Rev. Genet., 38, 233-271.

5. Raji,H. and Hartsuiker,E. (2006) Double-strand break repair and homologous recombination in Schizosaccharomyces pombe. Yeast, 23, 963-976.

6. Liu,J. and Heyer,W.-D. (2011) Who's who in human recombination: BRCA2 and RAD52. Proc. Natl. Acad. Sci. U.S.A., 108, 441-442.

7. Prakash,R., Zhang,Y., Feng,W. and Jasin,M. (2015) Homologous recombination and human health: the roles of BRCA1, BRCA2, and associated proteins. Cold Spring Harb. Perspect. Biol., 7, a016600.

8. Yuan,J. and Chen,J. (2011) The role of the human SWI5-MEI5 complex in homologous recombination repair. J. Biol. Chem., 286, 9888-9893.

9. Argunhan,B., Murayama,Y. and Iwasaki,H. (2017) The differentiated and conserved roles of Swi5-Sfr1 in homologous recombination. FEBS Lett., 591, 2035-2047.

10. Akamatsu,Y., Tsutsui,Y., Morishita,T., Siddique,M.S.P., Kurokawa,Y., Ikeguchi,M., Yamao,F., Arcangioli,B. and Iwasaki,H. (2007) Fission yeast Swi5/Sfr1 and Rhp55/Rhp57 differentially regulate Rhp51-dependent recombination outcomes. EMBO J., 26, $1352-1362$.

11. Martín,V., Chahwan,C., Gao,H., Blais,V., Wohlschlegel,J., Yates,J.R., McGowan,C.H. and Russell,P. (2006) Sws1 is a conserved regulator of homologous recombination in eukaryotic cells. EMBO J., 25, 2564-2574.

12. Dziadkowiec,D., Petters,E., Dyjankiewicz,A., Karpiński,P., Garcia,V., Watson,A.T. and Carr,A.M. (2009) The role of novel genes $\operatorname{rrp} 1(+)$ and $\operatorname{rrp} 2(+)$ in the repair of DNA damage in Schizosaccharomyces pombe. DNA Repair (Amst.)., 8, 627-636.

13. Dziadkowiec,D., Kramarz,K., Kanik,K., Wiśniewski,P. and Carr,A.M. (2013) Involvement of Schizosaccharomyces pombe rrp1+ 
and rrp2 + in the Srs2- and Swi5/Sfr1-dependent pathway in response to DNA damage and replication inhibition. Nucleic Acids Res., 41, 8196-8209.

14. Barg-Wojas,A., Muraszko,J., Kramarz,K., Schirmeisen,K., Baranowska,G., Carr,A.M. and Dziadkowiec,D. (2020) Schizosaccharomyces pombe DNA translocases Rrp1 and Rrp2 have distinct roles at centromeres and telomeres that ensure genome stability. J. Cell Sci., 133, jcs230193.

15. Wei,Y., Diao,L.-X., Lu,S., Wang,H.-T., Suo,F., Dong,M.-Q. and Du,L.-L. (2017) SUMO-targeted DNA translocase Rrp2 protects the genome from Top2-induced DNA damage. Mol. Cell, 66, 581-596.

16. Carr,A.M., Paek,A.L. and Weinert,T. (2011) DNA replication: failures and inverted fusions. Semin. Cell Dev. Biol., 22, 866-874.

17. Schlacher,K., Christ,N., Siaud,N., Egashira,A., Wu,H. and Jasin,M. (2011) Double-strand break repair-independent role for BRCA2 in blocking stalled replication fork degradation by MRE11. Cell, 145, $529-542$.

18. Hashimoto,Y., Ray Chaudhuri,A., Lopes,M. and Costanzo,V. (2010) Rad51 protects nascent DNA from Mre11-dependent degradation and promotes continuous DNA synthesis. Nat. Struct. Mol. Biol., 17, 1305-1311.

19. Zellweger,R., Dalcher,D., Mutreja,K., Berti,M., Schmid,J.A., Herrador,R., Vindigni,A. and Lopes,M. (2015) Rad51-mediated replication fork reversal is a global response to genotoxic treatments in human cells. J. Cell Biol., 208, 563-579.

20. Bugreev,D. V, Rossi,M.J. and Mazin,A.V. (2011) Cooperation of RAD51 and RAD54 in regression of a model replication fork Nucleic. Acids. Res., 39, 2153-2164.

21. Vujanovic,M., Krietsch,J., Raso,M.C., Terraneo,N., Zellweger,R., Schmid,J.A., Taglialatela,A., Huang,J.W., Holland,C.L., Zwicky,K. et al. (2017) Replication fork slowing and reversal upon DNA damage require PCNA polyubiquitination and ZRANB3 DNA translocase activity. Mol. Cell, 67, 882-890.

22. Achar,Y.J., Balogh,D., Neculai,D., Juhasz,S., Morocz,M., Gali,H., Dhe-Paganon,S., Venclovas, C. and Haracska,L. (2015) Human HLTF mediates postreplication repair by its HIRAN domain-dependent replication fork remodelling. Nucleic. Acids. Res., 43, 10277-10291.

23. Kile,A.C., Chavez,D.A., Bacal,J., Eldirany,S., Korzhnev,D.M., Bezsonova,I., Eichman,B.F. and Cimprich,K.A. (2015) HLTF's ancient HIRAN domain binds $3^{\prime}$ DNA ends to drive replication fork reversal. Mol. Cell, 58, 1090-1100.

24. Bétous,R., Mason,A.C., Rambo,R.P., Bansbach,C.E., Badu-Nkansah,A., Sirbu,B.M., Eichman,B.F. and Cortez,D. (2012) SMARCAL1 catalyzes fork regression and Holliday junction migration to maintain genome stability during DNA replication. Genes Dev., 26, 151-162.

25. Bhat,K.P. and Cortez,D. (2018) RPA and RAD51: fork reversal, fork protection, and genome stability. Nat. Struct. Mol. Biol., 25, 446-453.

26. McGlynn,P. and Lloyd,R.G. (2002) Recombinational repair and restart of damaged replication forks. Nat. Rev. Mol. Cell Biol., 3, 859-870.

27. Lambert,S., Watson,A.T., Sheedy,D.M., Martin,B. and Carr,A.M. (2005) Gross chromosomal rearrangements and elevated recombination at an inducible site-specific replication fork barrier. Cell, 121, 689-702.

28. Pepe,A. and West,S.C. (2014) MUS81-EME2 promotes replication fork restart. Cell Rep., 7, 1048-1055.

29. Sung,P. and Klein,H.L. (2006) Mechanism of homologous recombination: mediators and helicases take on regulatory functions. Nat. Rev. Mol. Cell Biol., 7, 739-750.

30. Heyer,W.-D. (2015) Regulation of recombination and genomic maintenance. Cold Spring Harb. Perspect. Med., 7, a016501.

31. Dungrawala,H., Bhat,K.P., Le Meur,R., Chazin,W.J., Ding,X., Sharan,S.K., Wessel,S.R., Sathe,A.A., Zhao,R. and Cortez,D. (2017) RADX promotes genome stability and modulates chemosensitivity by regulating RAD51 at replication forks. Mol. Cell, 67, 374-386.

32. Bhat,K.P., Krishnamoorthy,A., Dungrawala,H., Garcin,E.B., Modesti,M. and Cortez,D. (2018) RADX modulates RAD51 activity to control replication fork protection. Cell Rep., 24, 538-545.

33. Wright,W.D. and Heyer,W.-D. (2014) Rad54 functions as a heteroduplex DNA pump modulated by Its DNA substrates and Rad51 during D loop formation. Mol. Cell, 53, 420-432.
34. Solinger,J.A., Kiianitsa,K. and Heyer,W.-D. (2002) Rad54, a Swi2/Snf2-like recombinational repair protein, disassembles Rad51:dsDNA filaments. Mol. Cell, 10, 1175-1188.

35. Mason,J.M., Dusad,K., Wright,W.D., Grubb,J., Budke,B., Heyer,W.-D., Connell,P.P., Weichselbaum,R.R. and Bishop,D.K. (2015) RAD54 family translocases counter genotoxic effects of RAD51 in human tumor cells. Nucleic. Acids. Res., 43, 3180-3196.

36. Spies,J., Waizenegger,A., Barton,O., Sürder,M., Wright,W.D., Heyer,W.-D. and Löbrich,M. (2016) Nek1 Regulates Rad54 to orchestrate homologous recombination and replication fork stability. Mol. Cell, 62, 903-917.

37. Piwko,W., Mlejnkova,L.J., Mutreja,K., Ranjha,L., Stafa,D. Smirnov,A., Brodersen,M.M., Zellweger,R., Sturzenegger,A. Janscak,P. et al. (2016) The MMS22L-TONSL heterodimer directly promotes RAD51-dependent recombination upon replication stress. EMBO J., 35, 2584-2601.

38. Chi,P., Kwon,Y., Visnapuu,M.-L., Lam,I., Santa Maria,S.R., Zheng,X., Epshtein,A., Greene,E.C., Sung,P. and Klein,H.L. (2011) Analyses of the yeast Rad51 recombinase A265V mutant reveal different in vivo roles of Swi2-like factors. Nucleic. Acids. Res., 39, 6511-6522.

39. Shah,P.P., Zheng,X., Epshtein,A., Carey,J.N., Bishop,D.K. and Klein,H.L. (2010) Swi2/Snf2-related translocases prevent accumulation of toxic Rad51 complexes during mitotic growth. Mol. Cell, 39, 862-872.

40. Chi,P., Kwon,Y., Seong,C., Epshtein,A., Lam,I., Sung,P. and Klein,H.L. (2006) Yeast recombination factor Rdh54 functionally interacts with the Rad51 recombinase and catalyzes Rad51 removal from DNA. J. Biol. Chem., 281, 26268-26279.

41. Sung,P. and Robberson,D.L. (1995) DNA strand exchange mediated by a RAD51-ssDNA nucleoprotein filament with polarity opposite to that of RecA. Cell, 82, 453-461.

42. Bennett,B.T. and Knight,K.L. (2005) Cellular localization of human $\operatorname{Rad} 51 \mathrm{C}$ and regulation of ubiquitin-mediated proteolysis of Rad51. J. Cell. Biochem., 96, 1095-1109.

43. Inano,S., Sato,K., Katsuki,Y., Kobayashi,W., Tanaka,H., Nakajima,K., Nakada,S., Miyoshi,H., Knies,K., Takaori-Kondo,A. et al. (2017) RFWD3-mediated ubiquitination promotes timely removal of both RPA and RAD51 from DNA damage sites to facilitate homologous recombination. Mol. Cell, 66, 622-634.

44. Tsutsui,Y., Kurokawa,Y., Ito,K., Siddique,M.S.P., Kawano,Y., Yamao,F. and Iwasaki,H. (2014) Multiple regulation of Rad51-mediated homologous recombination by fission yeast Fbh1. PLos Genet., 10, e1004542.

45. Chu,W.K., Payne,M.J., Beli,P., Hanada,K., Choudhary,C. and Hickson,I.D. (2015) FBH1 influences DNA replication fork stability and homologous recombination through ubiquitylation of RAD51. Nat. Commun., 6, 5931

46. Moreno,S., Klar,A. and Nurse,P. (1991) Molecular genetic analysis of fission yeast Schizosaccharomyces pombe. Methods Enzymol., 194, $795-823$.

47. Tsang,E., Miyabe,I., Iraqui,I., Zheng,J., Lambert,S. and Carr,A.M. (2014) The extent of error-prone replication restart by homologous recombination is controlled by Exo1 and checkpoint proteins. J. Cell Sci., 127, 2983-2994.

48. Haruta,N., Kurokawa,Y., Murayama,Y., Akamatsu,Y., Unzai,S., Tsutsui,Y. and Iwasaki,H. (2006) The Swi5-Sfrl complex stimulates Rhp51/Rad51- and Dmc1-mediated DNA strand exchange in vitro. Nat. Struct. Mol. Biol., 13, 823-830.

49. Kurokawa,Y., Murayama,Y., Haruta-Takahashi,N., Urabe,I. and Iwasaki,H. (2008) Reconstitution of DNA strand exchange mediated by Rhp51 recombinase and two mediators. PLoS Biol., 6, e88.

50. Ito,K., Murayama,Y., Kurokawa,Y., Kanamaru,S., Kokabu,Y., Maki,T., Mikawa,T., Argunhan,B., Tsubouchi,H., Ikeguchi,M. et al. (2020) Real-time tracking reveals catalytic roles for the two DNA binding sites of Rad51. Nat. Commun., 11, 2950.

51. Kim,W.J., Lee,H., Park,E.J., Park,J.K. and Park,S.D. (2001) Gainand loss-of-function of Rhp51, a Rad51 homolog in fission yeast, reveals dissimilarities in chromosome integrity. Nucleic Acids Res., 29, 1724-1732.

52. Matsuo,Y., Sakane,I., Takizawa,Y., Takahashi,M. and Kurumizaka,H. (2006) Roles of the human Rad51 L1 and L2 loops in DNA binding. FEBS J., 273, 3148-3159. 
53. Bernstein,K.A., Reid,R.J.D., Sunjevaric,I., Demuth,K., Burgess,R.C. and Rothstein,R. (2011) The Shu complex, which contains Rad51 paralogues, promotes DNA repair through inhibition of the Srs2 anti-recombinase. Mol. Biol. Cell, 22, 1599-1607.

54. Lorenz,A., Osman,F., Folkyte,V., Sofueva,S. and Whitby,M.C. (2009) Fbh1 limits Rad51-dependent recombination at blocked replication forks. Mol. Cell. Biol., 29, 4742-4756.

55. Ouyang,K.J., Woo,L.L., Zhu,J., Huo,D., Matunis,M.J. and Ellis,N.A (2009) SUMO modification regulates BLM and RAD51 interaction at damaged replication forks. PLoS Biol., 7, e1000252.

56. Afshar,N., Argunhan,B., Palihati,M., Taniguchi,G., Tsubouchi,H. and Iwasaki,H. (2021) A novel motif of Rad51 serves as an interaction hub for recombination auxiliary factors. Elife, 10, e64131.

57. Ronneberger,O., Baddeley,D., Scheipl,F., Verveer,P.J., Burkhardt,H., Cremer,C., Fahrmeir,L., Cremer,T. and Joffe,B. (2008) Spatial quantitative analysis of fluorescently labeled nuclear structures: Problems, methods, pitfalls. Chromosom. Res., 16, 523-562.

58. Nakamura,K., Okamoto,A., Katou, Y., Yadani,C., Shitanda,T., Kaweeteerawat,C., Takahashi,T.S., Itoh,T., Shirahige,K., Masukata,H. et al. (2008) Rad51 suppresses gross chromosomal rearrangement at centromere in Schizosaccharomyces pombe. EMBO J., 27, 3036-3046.

59. Teixeira-Silva,A., Ait Saada,A., Hardy,J., Iraqui,I., Nocente,M.C., Fréon,K. and Lambert,S. (2017) The end-joining factor Ku acts in the end-resection of double strand break-free arrested replication forks. Nat. Commun., 8, 1982.

60. Kramarz,K., Schirmeisen,K., Boucherit,V., Ait Saada,A., Lovo,C., Palancade,B., Freudenreich,C.H. and Lambert,S. (2020) The nuclear pore primes recombination-dependent DNA synthesis at arrested forks by promoting SUMO removal. Nat. Commun., 11, 5643.

61. Lock,A., Rutherford,K., Harris,M.A., Hayles,J., Oliver,S.G., Bähler,J. and Wood,V. (2019) PomBase 2018: user-driven reimplementation of the fission yeast database provides rapid and intuitive access to diverse, interconnected information. Nucleic Acids Res., 47, D821-D827.

62. Zaitseva,E.M., Zaitsev,E.N. and Kowalczykowski,S.C. (1999) The DNA binding properties of Saccharomyces cerevisiae Rad51 protein. J. Biol. Chem., 274, 2907-2915.

63. Amunugama,R., He,Y., Willcox,S., Forties,R.A., Shim,K.-S., Bundschuh,R., Luo,Y., Griffith,J. and Fishel,R. (2012) RAD51 protein ATP cap regulates nucleoprotein filament stability. J. Biol. Chem., 287, 8724-8736.

64. Li,X., Zhang,X.-P., Solinger,J.A., Kiianitsa,K., Yu,X., Egelman,E.H. and Heyer,W.-D. (2007) Rad51 and Rad54 ATPase activities are both required to modulate Rad51-dsDNA filament dynamics. Nucleic. Acids. Res., 35, $4124-4140$.

65. Flaus,A. and Owen-Hughes, T. (2011) Mechanisms for ATP-dependent chromatin remodelling: The means to the end. FEBS J., 278, 3579-3595.

66. Prasad,P. and Ekwall,K. (2013) A snapshot of Snf2 enzymes in fission yeast. Biochem. Soc. Trans., 41, 1640-1647.

67. Glickman,M.H. and Ciechanover,A. (2002) The ubiquitin-proteasome proteolytic pathway: destruction for the sake of construction. Physiol. Rev., 82, 373-428.

68. Onaka,A.T., Toyofuku,N., Inoue,T., Okita,A.K., Sagawa,M., Su,J., Shitanda,T., Matsuyama,R., Zafar,F., Takahashi,T.S. et al. (2016) Rad51 and Rad54 promote noncrossover recombination between centromere repeats on the same chromatid to prevent isochromosome formation. Nucleic. Acids. Res., 44, 10744-10757.

69. Unk,I., Hajdu,I., Blastyak,A. and Haracska,L. (2010) Role of yeast Rad5 and its human orthologs, HLTF and SHPRH in DNA damage tolerance. DNA Repair (Amst. )., 9, 257-267.

70. Brühl,J., Trautwein,J., Schäfer,A., Linne,U. and Bouazoune,K. (2019) The DNA repair protein SHPRH is a nucleosome-stimulated ATPase and a nucleosome-E3 ubiquitin ligase. Epigenetics Chromatin, 12, 52.

71. Maacke,H., Jost,K., Opitz,S., Miska,S., Yuan,Y., Hasselbach,L., Lüttges,J., Kalthoff,H. and Stürzbecher,H.-W. (2000) DNA repair and recombination factor Rad51 is over-expressed in human pancreatic adenocarcinoma. Oncogene, 19, 2791-2795.

72. Maacke,H., Opitz,S., Jost,K., Hamdorf,W., Henning,W., Krüger,S., Feller,A.C., Lopens,A., Diedrich,K., Schwinger,E. et al. (2000) Over-expression of wild-type Rad51 correlates with histological grading of invasive ductal breast cancer. Int. J. Cancer, 88, 907-913.

73. Hansen,L.T., Lundin,C., Spang-Thomsen,M., Petersen,L.N. and Helleday,T. (2003) The role of RAD51 in etoposide (VP16) resistance in small cell lung cancer. Int. J. Cancer, 105, 472-479.

74. Raderschall,E., Stout,K., Freier,S., Suckow,V., Schweiger,S. and Haaf,T. (2002) Elevated levels of Rad51 recombination protein in tumor cells. Cancer Res., 62, 219-225.

75. Tennstedt,P., Fresow,R., Simon,R., Marx,A., Terracciano,L., Petersen,C., Sauter,G., Dikomey,E. and Borgmann,K. (2013) RAD51 overexpression is a negative prognostic marker for colorectal adenocarcinoma. Int. J. Cancer, 132, 2118-2126.

76. Brown,E.T. and Holt,J.T. (2009) Rad51 overexpression rescues radiation resistance in BRCA2-defective cancer cells. Mol. Carcinog., 48, 105-109. 